\title{
On analytic bootstrap for interface and boundary CFT
}

\section{Parijat Dey and Alexander Söderberg}

Department of Physics and Astronomy, Uppsala University, Box 516, SE-751 20 Uppsala, Sweden

E-mail: parijat.dey@physics.uu.se, alexander.soderberg@physics.uu.se

ABSTRACT: We use analytic bootstrap techniques for a CFT with an interface or a boundary. Exploiting the analytic structure of the bulk and boundary conformal blocks we extract the CFT data. We further constrain the CFT data by applying the equation of motion to the boundary operator expansion. The method presented in this paper is general, and it is illustrated in the context of perturbative Wilson-Fisher theories. In particular, we find constraints on the OPE coefficients for the interface CFT in $4-\epsilon$ dimensions (upto order $\mathcal{O}\left(\epsilon^{2}\right)$ ) with $\phi^{4}$-interactions in the bulk. We also compute the corresponding coefficients for the non-unitary $\phi^{3}$-theory in $6-\epsilon$ dimensions in the presence of a conformal boundary equipped with either Dirichlet or Neumann boundary conditions upto order $\mathcal{O}(\epsilon)$, or an interface upto order $\mathcal{O}(\sqrt{\epsilon})$.

KEywords: Boundary Quantum Field Theory, Conformal Field Theory, Conformal and W Symmetry

ArXiv EPrint: 2012.11344 


\section{Contents}

1 Introduction 1

2 Analytic structure of the conformal blocks 3

2.1 Methodology 6

2.2 Bulk OPE coefficients 8

3 An interface CFT in $4-\epsilon$ dimensions $\quad 9$

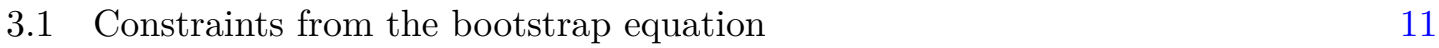

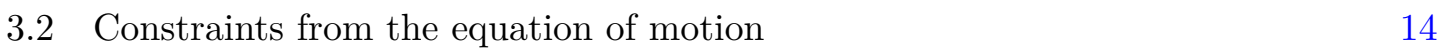

$\begin{array}{lll}3.3 & \text { CFT data at order } \epsilon^{2} & 17\end{array}$

$\begin{array}{lll}3.4 & \text { The renormalization group domain wall } & 17\end{array}$

4 A boundary CFT in $6-\epsilon$ dimensions $\quad 18$

$5 \quad$ An interface CFT in $6-\epsilon$ dimensions $\quad 22$

6 Conclusions 23

$\begin{array}{ll}\text { A Boundary conditions in an ICFT } & 24\end{array}$

B The $\epsilon$-expansion of boundary conformal blocks $\quad 25$

C Resummation techniques $\quad 26$

$\begin{array}{ll}\text { C.1 Expansion in } \xi & 26\end{array}$

$\begin{array}{lll}\text { C.2 Differentiate with respect to } \xi \text { and simplify using an ansatz } & 27\end{array}$

\section{Introduction}

Conformal field theories (CFTs) with boundaries or interfaces describe semi-infinite systems with differently-ordered regions. Boundaries and interfaces can be treated as codimension one defects. In a boundary CFT (BCFT), there is only a bulk theory on one side of the defect, with an unphysical region on the other side. In an interface CFT (ICFT), there is a bulk theory on each side of the codimension one defect. Interfaces can be realized physically in different ways. E.g. we can consider a quantum field theory (QFT) with two different vacua, with a finite energy barrier (that allows quantum tunneling) between them. This finite energy barrier can be described effectively by an interface, where the bulk theories correspond to the two vacua. Another realization is to probe a CFT with an operator from another CFT. We can then use the operator/state correspondence on this operator, which yields a small sphere around it. This sphere can be mapped to a line using 
a conformal transformation, which corresponds to an interface communicating with the two CFTs. An especially interesting case is when we probe a free theory with an interacting one, which ends up with the renormalization group (RG) domain wall studied in [1]. It has been speculated that such interface can tell us about the RG flow of the theory.

BCFTs and ICFTs have reduced symmetries compared to homogeneous CFTs (without a boundary or interface). However, they can still provide information about the bulk CFTs. These theories contain both the bulk operators as well as operators living on the boundary or the interface, and allow CFT techniques to be extended to a larger domain in the space of QFTs. The bulk CFT data, i.e. the spectrum of bulk operators and operator product expansion (OPE) coefficients, is a local property of the bulk CFT and is unaffected by the presence of the boundary or interface. BCFTs (ICFTs) are also characterized by the additional data: the spectrum of boundary (interface) operators and the boundary (interface) operator expansion (BOE/IOE) coefficients. Since the conformal symmetry is partially broken due to the presence of the boundary, the bulk operators can have nonvanishing one-point functions as a result of which the non-trivial observables in such systems are the two-point correlation function of bulk operators. These two-point functions can be expanded in two configurations. One can consider the case where both the operators are close to the boundary but far from each other. This is known as the boundary-channel expansion which involves the boundary operator dimensions and the BOE coefficients. On the other hand, the two-point function can be expanded in another channel where both the operators are close to each other but far from the boundary. This is known as the bulk-channel expansion and contains the bulk spectrum and OPE coefficients. A detailed analysis of BCFTs in this context can be found in [2]. The equality of the bulk- and boundary-channel results in a bootstrap equation for the BCFT that can be used to study the bulk and boundary data. This was initiated in [3]. Bootstrap techniques for BCFT and ICFT were studied in [1]. See [4-11] for recent works on BCFTs.

In perturbation theory where we have an expansion of the CFT data in terms of the perturbative parameter, the computation involves many Feynman diagrams at higher loop orders. One can simplify these computations by the bootstrap techniques to compute the BCFT/ICFT data with some inputs from the Feynman diagrams for the bulk CFT. The BCFT data in $4-\epsilon$ dimensions at the Wilson-Fisher (WF) fixed point was computed analytically upto $\mathcal{O}(\epsilon)$ in [3] using conformal bootstrap methods. This was further studied upto $\mathcal{O}\left(\epsilon^{2}\right)$ in [12] exploiting the analytic properties of the conformal blocks. One can also use analytic functionals to study BCFTs $[13,14]$. The approach in [12] makes use of the analytic structure of the branch cuts in the bulk- and boundary-channel to extract the CFT data, although it is more suited towards theories where only even or odd operators (w.r.t. the scaling dimensions at $\mathcal{O}\left(\epsilon^{0}\right)$ ) are present in the boundary-channel. We modify this method such that it yields more constraints on the CFT data in theories where both even and odd operators in the boundary-channel may appear.

The method we present consists of two parts. First we study the bootstrap equation and its analytic structure to constrain the CFT data. This is done completely without reference to the Lagrangian. In the second part we make use of the Lagrangian description of the system, in particular its equation of motion (e.o.m.), together with the BOE to 
further constrain the CFT data. This is similar to the method in [15], which has also been applied to the $\mathbb{Z}_{2^{-}}$and $O(N)$-twist defect $[16,17]$, and more recently to BCFTs [18]. We find that in the free theory of any unitary scalar CFT with a Lagrangian description, only the fundamental scalar and its normal derivative may appear in the BOE. We proceed to consider a BCFT in $3-\epsilon$ dimensions as well as a BCFT and ICFT in $4-\epsilon$ dimensions, and expand in $\epsilon$ to find the anomalous dimension for the boundary operators from the free theory.

The primary model we study is the CFT with an interface near four dimensions. We focus on the two-point correlation function of bulk scalar operators. From the bootstrap equation we impose constraints on the CFT data. We compute the correlator by resumming this data. Then we impose the e.o.m. on the correlator to further constrain the CFT data. The constraints we find are summarised in subsection 3.3. In the limit when one side of the interface is free, we make contact with the RG domain wall [1]. Finally we explore the CFT with a cubic interaction near six dimensions in the presence of a boundary subject to Dirichlet or Neumann boundary conditions (b.c.'s), or an interface.

The paper is organised as follows. In section 2 we discuss the analytic structure of the conformal blocks and how these can be used to analyze the bulk and boundary data. We discuss the entire methodology that we use to constrain the CFT data with a discussion on the similarities and differences with [12]. We also find a general expression for the bulk OPE coefficients (2.18) in $d$ dimensions at order $\epsilon^{k}$ in terms of the anomalous dimensions as well as the OPE coefficients from the previous order in the expansion parameter. In section 3 we study the bootstrap constraints on the two-point correlator of scalar operators in $4-\epsilon$ dimensions in an ICFT with scalars on both sides of the interface, transforming in the fundamental representation of $O(N) \times O(N)$. This is followed by further constraints on the data from the e.o.m.. In section 4 we bootstrap the scalar correlator for $\phi^{3}$-theory in $6-\epsilon$ dimensions in a BCFT with Dirichlet/Neumann b.c.. Section 5 contains the study of an ICFT in $6-\epsilon$ dimensions. We conclude in section 6 with some open questions and future directions. The appendices give the calculational details.

\section{Analytic structure of the conformal blocks}

In this section we discuss the analytic structure of the conformal blocks in a CFT with a boundary. We consider a BCFT defined in a $d$-dimensional semi-infinite space

$\mathbb{R}_{+}^{d}=\left\{x=\left(x_{\|}, z\right): x_{\|} \in \mathbb{R}^{d-1}, z>0\right\}$ bounded by a flat $(d-1)$-dimensional hypersurface at $z=0$. Due to the boundary at $z=0$ the translational invariance along the $z$-direction is broken whereas this invariance is preserved in the $x_{\|}$-direction.

We will consider scalar theories with $O(N)$-symmetry, and assume that there are no boundary effects that break this global symmetry (e.g. by an external field or terms quadratic in the fields on the boundary/interface action). If the $O(N)$-symmetry is explicitly broken, which would yield non-zero one-point functions for the scalar, the problem is more complex and requires more advanced methods [6, 19]. I.e. if the breaking is due to quadratic boundary-terms, then the bulk one-point function is non-zero at the bulk critical point if these interacting boundary conditions favour order at the boundary/interface. 
The two-point correlation function of two bulk scalar operators $\phi$ with scaling dimension $\Delta_{\phi}$ is given by

$$
\langle\phi(x) \phi(y)\rangle=\frac{F(\xi)}{|x-y|^{2 \Delta_{\phi}}},
$$

where the cross-ratio $\xi$ reads

$$
\xi=\frac{s_{\|}^{2}+\left(z-z^{\prime}\right)^{2}}{4 z z^{\prime}}, \quad s_{\|}^{a} \equiv x_{\|}^{a}-y_{\|}^{a}, \quad a \in\{1, \ldots, d-1\} .
$$

The two-point function can be decomposed in the bulk- or boundary-channel

$$
F(\xi)=\sum_{\Delta \geq 0} \lambda a_{\Delta} \mathcal{G}_{\text {ope }}(\Delta ; \xi)=\xi^{\Delta_{\phi}} \sum_{\hat{\Delta} \geq 0} \mu_{\hat{\Delta}}^{2} \mathcal{G}_{\text {boe }}(\hat{\Delta} ; \xi),
$$

where the conformal blocks are given by [2]

$$
\begin{aligned}
& \mathcal{G}_{\text {ope }}(\Delta ; \xi)=\xi^{\Delta / 2}{ }_{2} F_{1}\left(\frac{\Delta}{2}, \frac{\Delta}{2} ; \Delta+1-\frac{d}{2} ;-\xi\right) \\
& \mathcal{G}_{\text {boe }}(\hat{\Delta} ; \xi)=\xi^{-\hat{\Delta}}{ }_{2} F_{1}\left(\hat{\Delta}, \hat{\Delta}+1-\frac{d}{2} ; 2 \hat{\Delta}+2-d ;-\xi^{-1}\right) .
\end{aligned}
$$

In (2.3) the coefficients $\mu_{\hat{\Delta}}^{2}$ are the BOE coefficients squared and $\lambda a_{\Delta}$ are the bulk OPE coefficients times the one-point functions. The terms $\Delta=0$ as well as $\hat{\Delta}=0$ represent the contribution of bulk and boundary identity operators respectively.

Let us now look into the analytic structure of the bulk and boundary conformal blocks $\mathcal{G}_{\text {ope }}$ and $\mathcal{G}_{\text {boe }}$. Both of the blocks in (2.4) have a branch cut at $\xi<0$ that originates from generic non-integer power of $\xi$. There is an additional branch cut at $\xi<-1$ in the bulk block from the hypergeometric function with argument $-\xi$. The hypergeometric function in the boundary block with argument $-\xi^{-1}$ has a branch cut at $\xi \in(-1,0)$. This analytic structure has some important consequences as discussed in [12]. Let us define the discontinuity of a function $f(\xi)$ as

$$
\operatorname{disc}_{\xi} f(\xi) \equiv \lim _{\alpha \rightarrow 0^{+}} f(\xi+i \alpha)-f(\xi-i \alpha) .
$$

If we take the discontinuity of the bootstrap equation (2.3) at $\xi<-1$ the boundary blocks with $\hat{\Delta}=\frac{d-2}{2}+m, m \in \mathbb{Z}_{\geq 0}$ disappear from the equation (this holds for any $d$ ). These boundary operators correspond to normal derivatives, $\partial_{\perp}^{m} \hat{\phi}$, of a scalar with scaling dimension $\frac{d-2}{2}$. Also, by taking the discontinuity of (2.3) at $-1<\xi<0$ we can remove all the bulk blocks with $\Delta=d-2+2 n, n \in \mathbb{Z}_{\geq 0}$. Such operators correspond to scalar double traces $\phi \partial^{2 n} \phi$ of a scalar with dimension $\frac{d-2}{2}$.

We can understand the convergence of the bootstrap equation (2.3) under this analytic continuation using the radial coordinates introduced in [20] for defect CFTs. There will be one radial coordinate for each bootstrap-channel. The one for the boundary-channel changes sign under this analytic continuation, and is still within the region of convergence. On the other hand, the radial coordinate in the bulk-channel approaches the boundary 

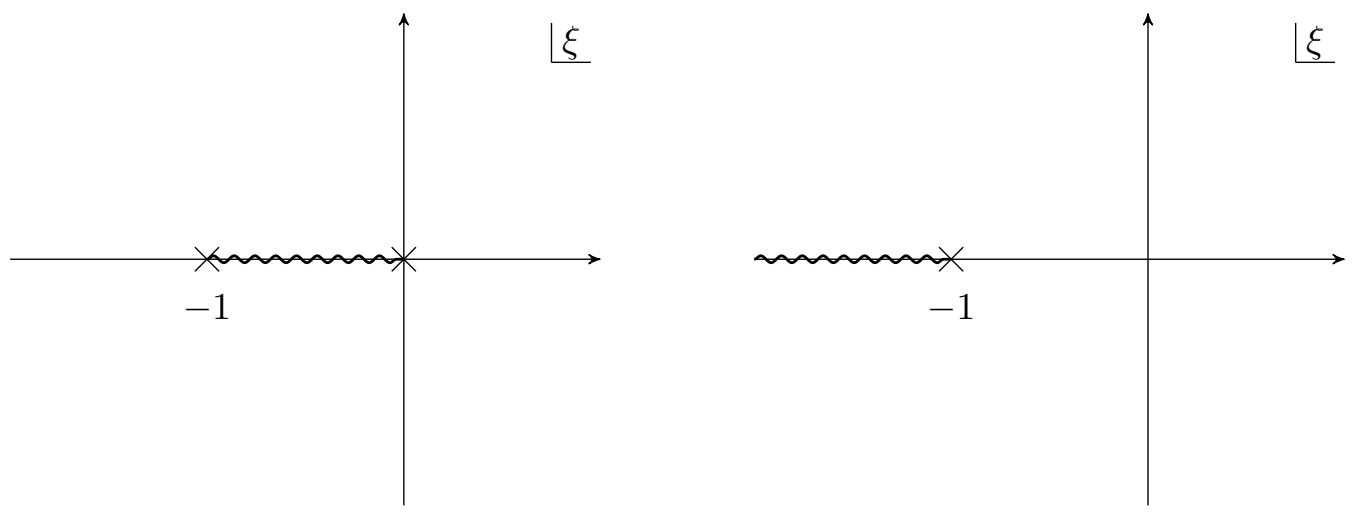

Figure 1. Analytic structure of the hypergeometric function in $\mathcal{G}_{\text {boe }}$ (left) and $\mathcal{G}_{\text {ope }}$ (right) in (2.4).

under this analytic continuation. This means that we should tread carefully and explicitly check that the bootstrap channels have the assumed branch cuts after resummation of the bulk OPE coefficients [12].

The discontinuity of the bulk block at $\xi<-1$ for the bulk exchange of double-trace operators of dimension $\Delta=d-2+2 n$ can be expressed in terms of Jacobi polynomials (this holds for any $d$ )

$$
\begin{aligned}
\underset{\xi<-1}{\operatorname{disc}} \mathcal{G}_{\text {ope }}(d-2+2 n ; \xi) & =2 \pi i(-1)^{\frac{d}{2}} \xi^{n+\frac{d}{2}-1} \frac{\Gamma_{2 n+\frac{d}{2}-1}}{\Gamma_{n}^{2} \Gamma_{\frac{d}{2}}}{ }_{2} F_{1}\left(\frac{d}{2}+n-1, \frac{d}{2}+n-1 ; \frac{d}{2} ; \xi+1\right) \\
& =-2 \pi i(-1)^{n} \frac{\Gamma_{2 n+\frac{d}{2}-1}}{\Gamma_{n} \Gamma_{n-1+\frac{d}{2}}} P_{n-1}^{\left(\frac{d}{2}-1,0\right)}\left(-\frac{\xi+2}{\xi}\right),
\end{aligned}
$$

where $\Gamma_{x} \equiv \Gamma(x)$ is the shorthand notation for the Gamma function and we have used the following identity

$$
{ }_{2} F_{1}(a, b, c, z)=\frac{1}{(1-z)^{a}}{ }_{2} F_{1}\left(a, c-b, c, \frac{z}{z-1}\right)
$$

in going from the first line to the second line of (2.6). The Jacobi polynomials satisfy the orthogonality relation

$$
\int_{-1}^{+1} d y(1-y)^{\frac{d}{2}-1} P_{m-1}^{\left(\frac{d}{2}-1,0\right)}(y) P_{n-1}^{\left(\frac{d}{2}-1,0\right)}(y)=\frac{\delta_{m n}}{2(m-1)+\frac{d}{2}}
$$

which holds for all $m, n \in \mathbb{Z}_{\geq 1}$.

Let us now look into the boundary blocks for the exchange operators of dimensions $\hat{\Delta}=\frac{d-2}{2}+n$. They satisfy the following orthogonality relation ${ }^{1}$

$$
\oint_{|w|=\tilde{\epsilon}} \frac{d w}{2 \pi i} w^{n-m-1}{ }_{2} F_{1}\left(1-m,-m-\frac{d-4}{2}, 2(1-m),-w\right){ }_{2} F_{1}\left(n, n+\frac{d-2}{2}, 2 n,-w\right)=\delta_{m, n},
$$

where $\tilde{\epsilon} \ll 1$.

\footnotetext{
${ }^{1}$ This can be found using an ansatz on the form $w^{n-m-1}{ }_{2} F_{1}\left(a_{1}+a_{2} m, a_{3}+a_{4} m, a_{5}+a_{6} m,-w\right)$ for the orthogonality weight function, where the coefficients $a_{i}, i \in\{1, \ldots, 6\}$, can be found by studying the residue at $w=0$ for different values of $m$ and $n$.
} 
The boundary conformal blocks have another analytical property that under the transformation $\xi \rightarrow e^{ \pm \pi i}(\xi+1)$ they acquire a phase $[12]^{2}$

$$
\mathcal{G}_{\text {boe }}\left(\hat{\Delta} ; e^{ \pm \pi i}(\xi+1)\right)=e^{\mp \pi i \hat{\Delta}} \mathcal{G}_{\text {boe }}(\hat{\Delta} ; \xi)
$$

In particular this means that for integer boundary dimensions $\hat{\Delta}=m$

$$
\mathcal{G}_{\text {boe }}(m ;-\xi-1)=(-1)^{m} \mathcal{G}_{\text {boe }}(m ; \xi)
$$

\subsection{Methodology}

In this subsection we discuss the general method adapted in this paper to extract the CFT data in section 3, 4 and 5. Throughout this paper we assume there exist a fundamental scalar, and that the exchanged operators are the scalar double traces $\phi \partial^{2 n} \phi$ in the bulk and the normal derivatives $\partial_{\perp}^{m} \hat{\phi}$ on the boundary. That is, in the free theory we have ${ }^{3}$

$$
\Delta_{n \geq 0}^{(\text {free })}=2\left(\Delta_{\phi}^{(\text {free })}+n\right), \quad \hat{\Delta}_{m \geq 0}^{(\text {free })}=\Delta_{\phi}^{(\text {free })}+m, \quad \Delta_{\phi}^{(\text {free })}=\frac{d-2}{2} .
$$

Moreover, we assume a two-point function and its corresponding CFT data is known (up to a finite set of free parameters) at some order in the expansion parameter, say $\mathcal{O}\left(\epsilon^{k-1}\right)$ for some $k \geq 1{ }^{4}$ We will denote operators multiplying the $\epsilon^{k}$-corrections of the OPE coefficients as new operators. Their anomalous dimensions contribute at order $\mathcal{O}\left(\epsilon^{k+1}\right)$. Thus at order $\mathcal{O}\left(\epsilon^{k}\right)$ it is enough to only consider their free scaling dimensions. If we assume these to be the ones above (for high enough $k$ this will generally not be true, and other operators will be exchanged as well), then their corresponding blocks satisfy the technology discussed in the previous subsection. Using the properties of new operators, the CFT data at $\mathcal{O}\left(\epsilon^{k}\right)$ can be found using our method.

The method we present is two-fold. Firstly we use the bootstrap equation perturbatively at each order in an expansion parameter to impose constraints on the CFT data. ${ }^{5}$ This is done completely without reference to a Lagrangian. Secondly, if we have a Lagrangian description of our system, we impose the e.o.m. on the correlator found from the bootstrap equation. This allows us to further constrain the CFT data of the model.We will outline the essential steps of the method below:

1. Finding constraints on the CFT data using the bootstrap method:

(a) Study the discontinuity of the bootstrap equation along $\xi<-1$, and assume that the discontinuity commutes with the series' of new operators. Due to the analytical structure of the conformal blocks, this assumes that the contribution from new operators in the bulk and boundary have a branch cut along $\xi<-1$

\footnotetext{
${ }^{2}$ This is the same as sending one of the external fields through the boundary $z \rightarrow-z$.

${ }^{3}$ This assumption on the exchanged scaling dimensions is motivated from the block decomposition of a generalized free scalar [3].

${ }^{4}$ E.g. in [3] the CFT data was fixed up to one free parameter at $\mathcal{O}(\epsilon)$ (without using information of the bulk theory as input).

${ }^{5}$ Such parameter could e.g. be the deviation from the integer spacetime dimension $\epsilon \ll 1$ or $N^{-1} \ll 1$ in $O(N)$-models. The former will be considered in this paper.
} 
and $\xi \in(-1,0)$ respectively. The contributions from new bulk OPE coefficients will be given in terms of the Jacobi polynomials (2.6). Using the orthogonality relation (2.8) for these polynomials we are then able to project out the bulk OPE coefficients. These will be given in terms of the anomalous dimensions of the exchanged operators at the previous order in the expansion parameter.

(b) Resum the bulk-channel and find the contribution from new operators on the boundary. As was discussed in the previous section, the resummed bulk-channel should only have a branch cut along $\xi<-1$. Regarding the contribution from new operators on the boundary, since we assumed that we could commute the discontinuity with the series' in step 1a, this contribution is not allowed to have branch cuts along $\xi<-1$. This means that we should set such terms to zero, which constrains the CFT data.

(c) If it is a BCFT, we can impose the b.c., e.g. Dirichlet or Neumann, to further constrain the CFT data. In particular, for Neumann b.c.'s when we study the boundary limit of $\partial_{\perp} \phi$ there may be a pole in $z$ which corresponds to the BOE exchange of $\hat{\phi}$ which we cannot set to zero. Instead the $z^{0}$-term should be set to zero, which is the contribution from the boundary operator $\partial_{\perp} \hat{\phi}$.

(d) We can project out BOE coefficients from the bootstrap equation using the orthogonality relation (2.9) for the boundary blocks. ${ }^{6}$

(e) The orthogonality relation in the previous step does not state which operators should appear in the BOE. We thus have to resum the new boundary blocks and check that it reproduces the original contribution.

Even and odd operators (w.r.t. the boundary scaling dimensions at the lowest order in the expansion parameter) should be resummed separately. These contributions are even or odd respectively under the transformation (2.11), which allows us to find more constraints on the CFT data. Using these constraints, the original contribution from new boundary blocks should equal the contributions from both even and odd operators.

2. Finding constraints on the CFT data using the e.o.m.:

(a) Impose the e.o.m. on the correlator found from step 1. This further constrains the CFT data. However, one has to be a bit careful since a problem with mixing may occur at higher orders in the expansion parameter.

The $\epsilon$-expansion of a BCFT in $4-\epsilon$ dimensions (with either Dirichlet or Neumann b.c.'s) was bootstrapped (upto order $\mathcal{O}\left(\epsilon^{2}\right)$ ) in [12]. The bootstrap method from that work differs slightly from the one presented here. Step $1 \mathrm{~b}$ and 1c were skipped completely, and only even (Neumann) or odd (Dirichlet) operators were assumed to be exchanged in the BOE. This means that we can use the transformation property (then called image symmetry) from step 1e to constrain the CFT data before finding the BOE coefficients in step 1d.

\footnotetext{
${ }^{6}$ Alternatively, we may decompose the contribution from new boundary operators in conformal blocks.
} 
This simplifies the calculations, although one cannot do this for theories where both even and odd operators appear. In this work we only consider such theories, where our method allows us to constrain the CFT data further.

The bootstrap method presented in this paper reproduces the result from [12] for Neumann b.c.'s. For Dirichlet b.c.'s, the transformation property in step 1e is trivially satisfied and hence we do not find as many constraints on the CFT data. This means that the method in [12] works better for theories where only even or odd operators appear, but not in the case when both even and odd operators appear.

Since both the discontinuity of the bulk block (2.6) as well as the orthogonality relation for the boundary block (2.9) hold in any spacetime dimension $d$, the method we present is independent of this parameter. This means that it can also be applied to e.g. threedimensional theories where the scaling dimensions are half-integers at the lowest order in the expansion parameter.

If there are infinitely many operators at the previous order in the expansion parameter, we need to use the bulk and boundary anomalous dimensions as input and resum their contribution before we can apply the method presented in this paper. In such case one also has to solve a mixing problem.

\subsection{Bulk OPE coefficients}

Here we will perform step 1a of the method presented in the previous subsection. This can be done generally for any spacetime dimension if we assume that the operators with scaling dimensions (2.12) are exchanged upto $\mathcal{O}\left(\epsilon^{k}\right)$. To illustrate this, assume that we know the correlator and its corresponding CFT data at order $\epsilon^{k-1}$ for some $k \in \mathbb{Z}_{\geq 1}$ (up to possibly some free parameters).

Scaling dimensions with a superscript (free) denotes the ones in (2.12) without anomalous dimensions. If they do not have this subscript, their anomalous dimensions upto $\mathcal{O}\left(\epsilon^{k}\right)$ is included as well. We expand the OPE coefficients in the following way ${ }^{7}$

$$
\begin{array}{cc}
\lambda a_{\mathbb{1}} & =\widetilde{\lambda a_{\mathbb{1}}}+\mathcal{O}\left(\epsilon^{k+1}\right), \\
\mu_{\mathbb{1}}^{2}=\tilde{\mu}_{\mathbb{1}}^{2}+\mathcal{O}\left(\epsilon^{k+1}\right), & \\
\lambda a_{\phi^{2}}=\widetilde{\lambda a_{0}}+\mathcal{O}\left(\epsilon^{k+1}\right), & \\
\lambda a_{\phi \partial^{2 n} \phi}=\widetilde{\lambda a_{n}}+\epsilon^{k} \lambda a_{n}^{(k)}+\mathcal{O}\left(\epsilon^{k+1}\right), & \text { if } n \in \mathbb{Z}_{[0, \tilde{n}]}, \\
\lambda a_{\phi \partial^{2 n} \phi}=\epsilon^{k} \lambda a_{n}^{(k)}+\mathcal{O}\left(\epsilon^{k+1}\right), & \text { if } n \in \mathbb{Z}_{>\tilde{n}}, \\
\mu_{\partial_{\perp}^{m} \phi}^{2}=\tilde{\mu}_{m}^{2}+\epsilon^{k} \mu_{m}^{(k)}+\mathcal{O}\left(\epsilon^{k+1}\right), & \text { if } m \in \mathbb{Z}_{[0, \tilde{m}]}, \\
\mu_{\partial_{\perp}^{m} \phi}^{2}=\epsilon^{k} \mu_{m}^{(k)}+\mathcal{O}\left(\epsilon^{k+1}\right), & \text { if } m \in \mathbb{Z}_{>\tilde{m}} .
\end{array}
$$

Here the CFT data with a tilde is that from order $\epsilon^{k-1}$, and $\tilde{n}, \tilde{m} \in \mathbb{Z}_{\geq 0}$ denotes the number of exchanged operators at order $\epsilon^{k-1}$. If infinitely many operators $(\tilde{m}, \tilde{n} \rightarrow+\infty)$ are exchanged at order $\epsilon^{k-1}$, we need to resum their contribution to the bootstrap equation

\footnotetext{
${ }^{7}$ In section 4 we find that near six dimensions we should assume a bulk $\phi$-exchange (instead of $\phi^{2}$ ) due to the e.o.m.. This will not affect the formula we find for the bulk OPE coefficients.
} 
before we can find the OPE coefficients at order $\epsilon^{k}$. This requires all of the corresponding anomalous dimensions as input.

We will write the bootstrap equation in terms of new operators (these are included in $H_{b}$ and $H_{i}$ )

$$
\begin{array}{cc}
F(\xi)=G_{b}(\xi)+H_{b}(\xi)+\mathcal{O}\left(\epsilon^{k+1}\right)=G_{i}(\xi)+H_{i}(\xi)+\mathcal{O}\left(\epsilon^{k+1}\right), \\
G_{b}=\widetilde{\lambda a_{\mathbb{1}}}+\sum_{n=0}^{\tilde{n}} \widetilde{\lambda a_{n}} \mathcal{G}_{\mathrm{ope}}\left(\Delta_{n} ; \xi\right), & H_{b}=\epsilon^{k} \sum_{n \geq 1} \lambda a_{n}^{(k)} \mathcal{G}_{\mathrm{ope}}\left(\Delta_{n}^{(\text {free })} ; \xi\right), \\
G_{i}=\tilde{\mu}_{\mathbb{1}}^{2} \xi^{\Delta_{\phi}}+\sum_{m=0}^{\tilde{m}} \tilde{\mu}_{m}^{2} \xi^{\Delta_{\phi}} \mathcal{G}_{\mathrm{boe}}\left(\hat{\Delta}_{m} ; \xi\right), & H_{i}=\epsilon^{k} \sum_{m \geq 0} \mu_{m}^{(k)} \xi^{\Delta_{\phi}^{(\text {free })}} \mathcal{G}_{\mathrm{boe}}\left(\hat{\Delta}_{m}^{(\text {free })} ; \xi\right),
\end{array}
$$

where $\Delta_{n}$ and $\hat{\Delta}_{m}$ are the full scaling dimensions of $\phi \partial^{2 n} \phi$ and $\partial_{\perp}^{m} \hat{\phi}$ respectively, including their anomalous dimensions. Since the orthogonality relation (2.8) does not hold for the bulk $\phi^{2}$-exchange, we let its corresponding block be in $G_{b}$. If we consider the discontinuity along $\xi<-1(2.6)$, and assume that it commute with the series in $H_{b}$ and $H_{i}$ we find

$$
\begin{aligned}
\operatorname{disc}_{\xi<-1} H_{b} & =\epsilon^{k} \sum_{n \geq 1} \lambda a_{n}^{(k)} \operatorname{disc}_{\xi<-1} \mathcal{G}_{\text {ope }}\left(\Delta_{n}^{(\text {free })} ; \xi\right) \\
& =-2 \pi i \epsilon^{k} \sum_{n \geq 1} \lambda a_{n}^{(k)}(-1)^{n} \frac{\Gamma_{2 n+\frac{d}{2}-1}}{\Gamma_{n} \Gamma_{n+\frac{d}{2}-1}} P_{n-1}^{\left(\frac{d}{2}-1,0\right)}\left(-\frac{\xi+2}{\xi}\right), \\
\operatorname{disc}_{\xi<-1} H_{i} & =\epsilon^{k} \sum_{m \geq 0} \mu_{m}^{(k)} \operatorname{disc}_{\xi<-1} \mathcal{G}_{\mathrm{boe}}\left(\hat{\Delta}_{m}^{(\text {free })} ; \xi\right)=0 .
\end{aligned}
$$

Using the orthogonality relation (2.8), we find the bulk OPE coefficients as an integral using the bootstrap equation (2.15)

$$
\lambda a_{n}^{(k)}=-\left.\frac{\Gamma_{n} \Gamma_{n+\frac{d}{2}-1}}{\pi i \epsilon^{k} 2^{\frac{d}{2}+1}(-1)^{n} \Gamma_{2 n+\frac{d}{2}-2}} \int_{-1}^{+1} d y(1-y)^{\frac{d}{2}-1} P_{n-1}^{\left(\frac{d}{2}-1,0\right)}(y) \operatorname{disc}_{\xi<-1}\left(G_{i}-G_{b}\right)\right|_{\xi=-\frac{2}{y+1}} .
$$

This formula holds for any spacetime dimension assuming the exchanged operators are $\phi \partial^{2 n} \phi$ and $\partial_{\perp}^{m} \hat{\phi} . G_{i}-G_{b}$ is the theory-dependent part. The rest of the formula is universal. The special case of this formula when $d=4$ was studied in [12] at order $\epsilon^{2}$.

\section{An interface CFT in $4-\epsilon$ dimensions}

In this section we will consider a flat conformal interface spanned along $\mathbb{R}^{d-1}$ in a CFT in flat space $\mathbb{R}^{d}$ with a $\phi^{4}$ bulk interaction in $d=4-\epsilon$ dimensions, and study the expansion in $\epsilon$. Let us place the interface at the coordinate $z=0$. The ICFT we consider has an $O(N) \times$ $O(N)$ global symmetry which comes from two scalars $\phi_{ \pm}^{i}, i \in\{1, \ldots, N\}$, transforming in the fundamental representation of $O(N)$, defined on each side of the interface:

$\mathbb{R}_{+}^{d}=\left\{\left(x_{\|}, z\right): x_{\|} \in \mathbb{R}^{d-1}, z>0\right\}$ and $\mathbb{R}_{-}^{d}=\left\{\left(x_{\|}, z\right): x_{\|} \in \mathbb{R}^{d-1}, z<0\right\}$. On the interface we identify the scalars and their normal derivatives with each other ${ }^{8}$

$$
\hat{\phi}_{-}^{i}=\hat{\phi}_{+}^{i} \equiv \hat{\phi}^{i}, \quad \partial_{\perp} \hat{\phi}_{-}^{i}=\partial_{\perp} \hat{\phi}_{+}^{i} \equiv \partial_{\perp} \hat{\phi}^{i} .
$$

\footnotetext{
${ }^{8}$ See appendix A for details regarding this b.c.
} 
Hatted operators are local fields on the interface that appear in the BOE/IOE of bulk fields. Even in the free theory there may be poles in these expansions coming from the interface limit, see e.g. [4] for a discussion on how the BOE describes mixing of renormalized fields in the boundary limit.

Above b.c. was also considered in [21], and it reproduces the correlators from [1] upto $\mathcal{O}(\epsilon)$ when one side of the interface is free. Interfaces with different b.c.'s were studied in [22-26]. In particular, they considered either Dirichlet or Neumann b.c.'s on each side of the interface (which were allowed to be different on the two sides of the interface). Since the b.c.'s determine the correlators, we can use them to classify the interface. This means that in this paper we consider another interface, and thus we expect the CFT data we find to differ from their results.

The folding trick "converts" an ICFT to a BCFT simply by shifting $z \rightarrow-z$ in $\hat{\phi}_{-}^{i}[27]$

$$
\hat{\phi}_{-}^{i}\left(x_{\|},-z\right)-\hat{\phi}_{+}^{i}\left(x_{\|}, z\right)=0, \quad \partial_{\perp} \hat{\phi}_{-}^{i}\left(x_{\|},-z\right)+\partial_{\perp} \hat{\phi}_{+}^{i}\left(x_{\|}, z\right)=0 .
$$

These are Neumann ("+") and Dirichlet ("-") b.c.'s for the linear combinations $\hat{\phi}_{+}^{i} \pm \hat{\phi}_{-}^{i}$. Let us define a scalar in the representation $(N \times 1) \oplus(1 \times N)$ of $O(N) \times O(N)$

$$
\Phi_{\alpha}^{i}\left(x_{\|}, z\right)=\phi_{-}^{i}\left(x_{\|},-z\right) \delta_{\alpha-}+\phi_{+}^{i}\left(x_{\|}, z\right) \delta_{\alpha+}, \quad \alpha= \pm \Leftrightarrow \vec{\Phi}=\left(\begin{array}{l}
\vec{\phi}_{-} \\
\vec{\phi}_{+}
\end{array}\right),
$$

and also define projectors $\Pi_{ \pm}$that project out the linear combinations that satisfy Dirichlet and Neumann b.c.'s

$$
\left(\Pi_{ \pm}\right)_{\alpha \beta}^{i j}=\frac{\delta^{i j}}{2}\left(\begin{array}{cc}
1 & \pm 1 \\
\pm 1 & 1
\end{array}\right)_{\alpha \beta}, \quad \Pi_{ \pm} \vec{\Phi}=\left(\begin{array}{c}
\vec{\phi}_{-} \pm \vec{\phi}_{+} \\
\pm \vec{\phi}_{-}+\vec{\phi}_{+}
\end{array}\right) .
$$

We find the two-point correlator of $\Phi_{\alpha}^{i}$ in the free theory using the method of images

$$
\begin{aligned}
\left\langle\Phi_{\alpha}^{i}\left(x_{\|}, z\right) \Phi_{\beta}^{j}\left(y_{\|}, z^{\prime}\right)\right\rangle= & \left\langle\Phi_{\alpha}^{i}\left(x_{\|}, z\right) \Phi_{\beta}^{j}\left(y_{\|}, z^{\prime}\right)\right\rangle_{H} \\
& +\chi_{\beta \gamma}^{j k}\left\langle\Phi_{\alpha}^{i}\left(x_{\|}, z\right) \Phi_{\gamma}^{k}\left(y_{\|},-z^{\prime}\right)\right\rangle_{H}, \\
\left\langle\Phi_{\alpha}^{i}\left(x_{\|}, z\right) \Phi_{\beta}^{j}\left(y_{\|}, z^{\prime}\right)\right\rangle_{H}= & \frac{\delta^{i j} \delta_{\alpha \beta}}{\left[\left(x_{\|}-y_{\|}\right)^{2}+\left(z-z^{\prime}\right)^{2}\right]^{\left(\Delta_{\alpha}+\Delta_{\beta}\right) / 2}} .
\end{aligned}
$$

Here $\Delta_{\alpha}$ is the scaling dimension of $\phi_{\alpha}^{i}$, the subscript $H$ denotes that it is the correlator in a homogeneous CFT, and $\chi_{\alpha \beta}^{i j}$ is the difference between the projectors, i.e. the difference between fields satisfying Neumann and Dirichlet b.c.'s

$$
\chi_{\alpha \beta}^{i j}=\left(\Pi_{+}\right)_{\alpha \beta}^{i j}-\left(\Pi_{-}\right)_{\alpha \beta}^{i j}=\delta^{i j}\left(\begin{array}{ll}
0 & 1 \\
1 & 0
\end{array}\right)_{\alpha \beta} .
$$

The correlator (3.5) can be written in terms of the cross-ratio $\xi$ in (2.1)

$$
\left\langle\Phi_{\alpha}^{i}(x) \Phi_{\beta}^{j}(y)\right\rangle=\frac{\delta^{i j} F_{\alpha \beta}(v)}{|x-y|^{\Delta_{\alpha}+\Delta_{\beta}}}, \quad F_{\alpha \beta}(v)=\left(\begin{array}{cc}
1 & v^{\Delta_{\alpha}+\Delta_{\beta}} \\
v^{\Delta_{\alpha}+\Delta_{\beta}} & 1
\end{array}\right)_{\alpha \beta},
$$


where $v$ is defined as

$$
v^{2}=\frac{\xi}{\xi+1} .
$$

The two-point functions in a BCFT with Neumann or Dirichlet b.c.'s are superpositions of these ICFT correlators: $F_{\mathrm{BCFT}}^{ \pm}=F_{++} \pm F_{+-} \cdot{ }^{9}$ Note that $F_{ \pm \pm}$corresponds to the $\phi_{ \pm}-\phi_{ \pm}$ two-point functions with fields on the same side of the interface, and that $F_{+-}=F_{-+}$ corresponds to the $\phi_{+}-\phi_{-}$two-point functions with fields on opposite sides of the interface. Since $\phi_{+}$and $\phi_{-}$are on different sides of the interface, there is no well defined bulk OPE between them. This means that $F_{+-}$does not have a well-defined bulk-channel, but only a boundary-channel decomposition. Only $F_{ \pm \pm}$satisfy the bootstrap equation (2.3).

Expressed in terms of $x$ and $y$, this correlator is on the same form $\left(|x-y|^{-\left(\Delta_{\alpha}+\Delta_{\beta}\right)}\right)$ as a two-point correlator in a homogeneous CFT, which is a consequence of the b.c.'s (3.1). At higher orders in the expansion parameter it will differ though.

We will specialize to the case when the two external operators are the fundamental scalar

$$
\Delta_{+}=\Delta_{-}=\Delta_{\phi}^{(\text {free })}=\frac{d-2}{2}
$$

The free theory decomposition is then

$$
\begin{aligned}
\lambda a_{\mathbb{1}} & =1, & \lambda a_{\Delta} & =0, \\
\mu_{\mathbb{1}}^{2} & =0, & \mu_{\hat{\Delta}}^{2} & =\delta_{\hat{\Delta}, \Delta_{\phi}}+\frac{\Delta_{\phi}}{2} \delta_{\hat{\Delta}, \Delta_{\phi}+1}, \\
\left(\mu_{\mathbb{1}}^{2}\right)_{ \pm} & =0, & \left(\mu_{\hat{\Delta}}^{2}\right)_{ \pm} & =\delta_{\hat{\Delta}, \Delta_{\phi}}-\frac{\Delta_{\phi}}{2} \delta_{\hat{\Delta}, \Delta_{\phi}+1} .
\end{aligned}
$$

The interface operators with scaling dimensions $\Delta_{\phi}$ and $\Delta_{\phi}+1$ correspond to $\hat{\phi}$ and $\partial_{\perp} \hat{\phi}$ respectively. We labelled the IOE coefficients from $F_{+-}$with a " \pm " subscript.

\subsection{Constraints from the bootstrap equation}

In this subsection we will find constraints on the CFT data by solving the bootstrap equation (2.3) in $d=4-\epsilon$ dimensions. We will consider ( $\phi^{4}$-)interactions only in the bulk, and assume that they are the same on both sides of the interface. This means that $F_{++}$ is the same as $F_{--}$at all orders in perturbation theory. We will assume that the coupling constants are proportional to $\epsilon$ at their RG fixed point.

We begin by expanding the CFT data in $\epsilon$

$$
\begin{gathered}
\Delta_{\phi}=\Delta_{\phi}^{(\text {free })}+\epsilon \gamma_{\phi}^{(1)}+\epsilon^{2} \gamma_{\phi}^{(2)}+\mathcal{O}\left(\epsilon^{3}\right), \\
\Delta_{n \geq 0}=2\left(\Delta_{\phi}^{(\text {free })}+n\right)+\epsilon \gamma_{n}^{(1)}+\epsilon^{2} \gamma_{n}^{(2)}+\mathcal{O}\left(\epsilon^{3}\right), \\
\hat{\Delta}_{m \geq 2}=\Delta_{\phi}^{(\text {free })}+m+\epsilon \hat{\gamma}_{m}^{(1)}+\epsilon^{2} \hat{\gamma}_{m}^{(2)}+\mathcal{O}\left(\epsilon^{3}\right), \\
\lambda a_{\mathbb{1}}=1, \quad \lambda a_{\Delta_{n}}=\epsilon \lambda a_{n}^{(1)}+\epsilon^{2} \lambda a_{n}^{(2)}+\mathcal{O}\left(\epsilon^{3}\right), \\
\mu_{\mathbb{1}}^{2}=0, \quad \mu_{\hat{\Delta}_{m}}^{2}=\delta_{m 0}+\frac{\Delta_{\phi}^{(\text {free })}}{2} \delta_{m 1}+\epsilon \mu_{m}^{(1)}+\epsilon^{2} \mu_{m}^{(2)}+\mathcal{O}\left(\epsilon^{3}\right) .
\end{gathered}
$$

\footnotetext{
${ }^{9}$ Using the results of this paper and [12], one can check that this holds upto $\mathcal{O}\left(\epsilon^{2}\right)$.
} 
Here $\Delta_{\phi}^{(\text {free) }}$ is given by (3.9). One can bootstrap the order $\epsilon$ terms by expanding in $\xi$ around zero which allows us to fix the CFT data up to two parameters $\alpha$ and $\beta$

$$
\begin{aligned}
\gamma_{\phi}^{(1)} & =0, & \hat{\gamma}_{1}^{(1)}=\hat{\gamma}_{0}^{(1)} & =-\alpha, \\
\lambda a_{n}^{(1)} & =(\alpha+\beta) \delta_{n, 0}+\frac{\alpha}{2} \delta_{n, 1}, & \mu_{m}^{(1)} & =\beta \delta_{m, 0}+\frac{\alpha-\beta}{2} \delta_{m, 1} .
\end{aligned}
$$

These constraints are similar to those found when bootstrapping a BCFT correlator at order $\epsilon$ [3], with one major difference: in this case $\alpha$ is not related to any of the bulk anomalous dimensions, i.e. we cannot use bulk CFT data as input to find the OPE coefficients and the anomalous dimensions of boundary fields. Moreover there is one more free parameter in the ICFT case.

Let us proceed with bootstrapping the correlator at order $\epsilon^{2}$ using the method described in section $2 .{ }^{10}$ The boundary conformal blocks $\mathcal{G}_{\text {boe }}\left(\hat{\Delta}_{0}, \xi\right)$ and $\mathcal{G}_{\text {boe }}\left(\hat{\Delta}_{1}, \xi\right)$ cannot be expanded using the Mathematica package HypExp [28] since it only expands hypergeometric functions with parameters linear terms in $\epsilon$. However, the same algorithm can still be used. ${ }^{11}$

Let us write the bootstrap equation in the following way

$$
F_{ \pm \pm}(\xi)=G_{b}(\xi)+H_{b}(\xi)+\mathcal{O}\left(\epsilon^{3}\right)=G_{i}(\xi)+H_{i}(\xi)+\mathcal{O}\left(\epsilon^{3}\right)
$$

where $H_{b}$ and $H_{i}$ contain the contributions from new operators

$$
\begin{aligned}
& G_{b}(\xi)=1+\left(\epsilon(\alpha+\beta)+\epsilon^{2} \lambda a_{0}^{(2)}\right) \mathcal{G}_{\text {ope }}\left(\Delta_{0} ; \xi\right)+\frac{\epsilon \alpha}{2} \mathcal{G}_{\text {ope }}\left(\Delta_{1} ; \xi\right) \\
& G_{i}(\xi)=(1+\epsilon \beta) \xi^{\Delta_{\phi}} \mathcal{G}_{\text {boe }}\left(\hat{\Delta}_{0} ; \xi\right)+\frac{\Delta_{\phi}^{(\text {free })}+\epsilon(\alpha-\beta)}{2} \xi^{\Delta_{\phi}} \mathcal{G}_{\text {boe }}\left(\hat{\Delta}_{1} ; \xi\right) \\
& H_{b}(\xi)=\epsilon^{2} \sum_{n \geq 1} \lambda a_{n}^{(2)} \mathcal{G}_{\text {ope }}(2(n+1) ; \xi) \\
& H_{i}(\xi)=\epsilon^{2} \sum_{m \geq 0} \mu_{m}^{(2)} \xi \mathcal{G}_{\text {boe }}(m+1 ; \xi) .
\end{aligned}
$$

We included the $\lambda a_{0}^{(2)}$-term in $G_{b}$ for a technical reason: the corresponding conformal block $\mathcal{G}_{\text {ope }}(2 ; \xi)$ has no branch cut along $\xi<-1 . G_{b}$ and $G_{i}$ have branch cuts along the entire negative axis $\xi<0$ due to the non-integer powers of $\xi$, while the summand of $H_{b}$ has a branch cut along $\xi<-1$ and the summand of $H_{i}$ has a branch cut along $\xi \in(-1,0)$. The branch cuts in $H_{b}$ and $H_{i}$ follow from the hypergeometric functions in the conformal blocks (2.3) (see figure 1). We will consider the discontinuity (2.6) at $\xi<-1$, and assume that it commutes with the series' in $H_{b}$ and $H_{i}(2.17)$. We can then use the orthogonality relation (2.8) to project out the bulk OPE coefficients (2.18)

$$
\begin{aligned}
\lambda a_{n}^{(2)} & =\left.\frac{\Gamma_{n} \Gamma_{n+2}}{4 \pi i(-1)^{n} \Gamma_{2 n+1}} \int_{-1}^{+1} d y(1-y) P_{n-1}^{(1,0)}(y) \underset{\xi<-1}{\operatorname{disc}}\left[G_{i}(\xi)-G_{b}(\xi)\right]\right|_{\xi=-\frac{2}{y+1}} \\
& =\frac{(-1)^{n}(n !)^{2}}{(2 n) !} \int_{-1}^{+1} d y P_{n-1}^{(1,0)}(y)\left\{A_{1}+(1-y)\left[A_{2}+A_{3} \log \left(\frac{2}{1+y}\right)+A_{4} \log \left(\frac{1-y}{1+y}\right)\right]\right\},
\end{aligned}
$$

\footnotetext{
${ }^{10}$ We cannot naively expand in $\xi$ since only the interface-channel will then contain $\log (\xi)$-terms.

${ }^{11}$ We explain this algorithm and write out the expansions for the boundary blocks in appendix B.
} 


$$
\begin{aligned}
& A_{1}=-\frac{2 \alpha \beta-(\alpha+\beta) \gamma_{0}^{(1)}+\alpha \gamma_{1}^{(1)}-\hat{\gamma}_{0}^{(2)}+\hat{\gamma}_{1}^{(2)}}{2}, \\
& A_{2}=-\frac{\alpha\left(1-6 \gamma_{1}^{(1)}\right)+4 \gamma_{\phi}^{(2)}-2\left(\hat{\gamma}_{0}^{(2)}+\hat{\gamma}_{1}^{(2)}\right)}{8} \\
& A_{3}=-\frac{\alpha \gamma_{1}^{(1)}}{4} \\
& A_{4}=-\frac{\alpha\left(2 \alpha-\gamma_{1}^{(1)}\right)}{4} .
\end{aligned}
$$

These integrals can be computed to give

$$
\begin{aligned}
\lambda a_{1}^{(2)} & =-\left(A_{1}+A_{2}+\frac{3}{2} A_{3}+A_{4}\right), \\
\lambda a_{n \geq 2}^{(2)} & =\frac{2(n !)^{2}}{(2 n) !}\left(\frac{(-1)^{n} A_{1}}{n}-\frac{2 A_{3}}{n^{2}-1}-\frac{2 A_{4}}{n\left(n-(-1)^{n}\right)}\right) .
\end{aligned}
$$

We can now proceed to resum the bulk-channel using the procedure in appendix C.1

$H_{b}=-\epsilon^{2}\left(\frac{\xi}{\xi+1}\left(A_{1} \log (\xi+1)-2\left(A_{2}+A_{3}\right)\right)-2 A_{3} \operatorname{Li}_{2}(-\xi)+2 A_{2} \log (\xi+1)+A_{4} \log (\xi+1)^{2}\right)$.

As we can see, $H_{b}$ has a branch cut along $\xi<-1$ and none along $\xi \in(-1,0)$, which coincides with our assumptions when we commuted the discontinuity with the series in $H_{b}$, see (2.17).

The $H_{i}$ we find from the bootstrap equation (3.15) contains terms with a branch cut along $\xi<-1$

$$
H_{i} \ni \alpha \frac{\gamma_{1}^{(1)}-1}{2}\left(\log (\xi+1)\left(\log \left(\frac{\xi}{\xi+1}\right)+\frac{\log (\xi+1)}{2}\right)+\operatorname{Li}_{2}(-\xi)\right) .
$$

These terms are not allowed since we assumed in (2.17) that it only has a branch cut along $\xi \in(-1,0)$. If we demand these problematic terms to vanish we find

$$
\alpha \gamma_{1}^{(1)}=\alpha .
$$

That is, either $\alpha=0$ or $\gamma_{1}^{(1)}=1$. The latter is indeed the correct anomalous dimension of $\phi^{j} \partial^{2} \phi^{j}$ [29]. We will assume (3.20) for generality, and remarkably this removes the dependence of $\gamma_{1}^{(1)}$ in the bootstrap equation. With this constraint at hand, we can project out the IOE coefficients using the orthogonality relation (2.9)

$$
\begin{gathered}
\mu_{m}^{(2)}=\oint_{|w|=\tilde{\epsilon}} \frac{d w}{2 \pi i} \frac{{ }_{2} F_{1}(1-m,-m, 2(1-m),-w)}{w^{m+1}}\left(\frac{B_{1}}{w+1}+\frac{w B_{2}}{w+1}+\right. \\
\left.+\left(B_{3}+\frac{B_{4}}{w+1}\right) \log (w+1)+B_{5}\left[\log (w+1)^{2}+2 \operatorname{Li}_{2}(-w)\right]\right) . \\
B_{1}=-\frac{2 \gamma_{\phi}^{(2)}-\hat{\gamma}_{0}^{(2)}-\hat{\gamma}_{1}^{(2)}-2 \lambda a_{0}^{(2)}}{2}, \quad B_{2}=-\frac{(2 \alpha-\beta)(1-2 \alpha)+2 \hat{\gamma}_{1}^{(2)}}{4}, \\
B_{3}=\gamma_{\phi}^{(2)}, \quad B_{4}=\frac{\alpha(1+2 \beta)-(\alpha+\beta) \gamma_{0}^{(1)}-\hat{\gamma}_{0}^{(2)}+\hat{\gamma}_{1}^{(2)}}{2}, \quad B_{5}=-\frac{\alpha(1-2 \alpha)}{4} .
\end{gathered}
$$


These integrals can be performed which result in the following

$$
\begin{aligned}
\mu_{0}^{(2)} & =B_{1} \\
\mu_{1}^{(2)} & =-B_{1}+B_{2}+B_{3}+B_{4}-2 B_{5} \\
\mu_{m \geq 2}^{(2)} & =\frac{(m-2) !}{2^{2 m-3}(3 / 2)_{m-2}}\left(B_{3}-(-1)^{m} B_{4}-\frac{2(-1)^{m} B_{5}}{m(m-1)}\right),
\end{aligned}
$$

where $(a)_{b}=\frac{\Gamma(a+b)}{\Gamma(b)}$ is the Pochhammer symbol. We can proceed to resum even and odd operators separately using the procedure explained in appendix C.2. This yields two functions: $H_{i}^{+}$and $H_{i}^{-}$which corresponds to even and odd boundary operators respectively. Both of these satisfy the transformation property (2.11) on their own

$$
H_{i}(\xi)=H_{i}^{+}(\xi)+H_{i}^{-}(\xi),\left.\quad \frac{H_{i}^{ \pm}}{\xi}\right|_{\xi \rightarrow-(\xi+1)}= \pm \frac{H_{i}^{ \pm}}{\xi} .
$$

This property is trivially satisfied for even operators $\left(H_{i}^{+}\right)$while it yields a constraint on the CFT data for odd operators $\left(H_{i}^{-}\right)$

$$
B_{1}=0
$$

This constraint together with (3.18), (3.20) and (3.23) are the constraints we find from the bootstrap equation (2.3). They allow us to express all of the OPE coefficients in terms of the anomalous dimensions and the free parameters $\alpha$ and $\beta$.

\subsection{Constraints from the equation of motion}

Let us now apply the e.o.m. to the BOE (or IOE), and study how we can constrain the CFT data from the previous subsection further. We will prove that only $\hat{\phi}$ and $\partial_{\perp} \hat{\phi}$ may appear in a free scalar theory of any unitary BCFT with a Lagrangian description. At $\mathcal{O}(\epsilon)$ we find the anomalous dimensions of these operators. We will not explore beyond $\mathcal{O}(\epsilon)$ in this section due to mixing that will occur at $\mathcal{O}\left(\epsilon^{2}\right)$. This is done in a similar way as in [18].

The method we present is quite general, so we will not only do it for the ICFT we are considering, but also for a BCFT near four and three dimensions satisfying either Neumann or Dirichlet b.c.'s. See table 1 for our final result on the anomalous dimensions. We were not able to constrain the anomalous dimensions in the $\phi^{3}$-theory near six dimensions in this way.

The BOE of $\phi$ is given by [30]

$$
\phi^{i}(x)=\sum_{\hat{O}} \sum_{m \geq 0} \frac{\mu_{\hat{\Delta}} a_{\hat{\Delta}, m}}{|z|^{\Delta_{\phi}-\hat{\Delta}-2 m}} \partial_{\|}^{2 m} \hat{O}^{i}\left(x_{\|}\right), \quad a_{\hat{\Delta} m}=\frac{(-1)^{m}}{4^{m} m !\left(\hat{\Delta}-\frac{d-3}{2}\right)_{m}} .
$$

Here the BOE coefficients $\mu^{\phi^{i}} \hat{O}^{j} \equiv \mu_{\hat{\Delta}} \delta^{i j}$ are to be treated as $O(N)$-matrices, which we have assumed to be proportional to the identity. The BOE yields the following 
two-point function

$$
\begin{aligned}
\left\langle\phi^{i}(x) \hat{\phi}^{j}(y)\right\rangle & =\delta^{i j} \mu_{\hat{\Delta}} \sum_{m \geq 0} \frac{a_{\hat{\Delta} m}}{|z|^{\Delta_{\phi}-\hat{\Delta}-2 m}} \partial_{\|}^{2 m} \frac{A_{d}}{\left|s_{\|}\right|^{2 \hat{\Delta}}}, \\
A_{d} & =\frac{1}{(d-2) S_{d}}, \quad S_{d}=\frac{2 \pi^{d / 2}}{\Gamma_{d / 2}},
\end{aligned}
$$

where $S_{d}$ is the area of a $(d-1)$-dimensional sphere. We assumed the boundary fields live in an orthogonal basis

$$
\left\langle\hat{O}_{1}^{i}\left(x_{\|}\right) \hat{O}_{2}^{j}\left(y_{\|}\right)\right\rangle=\frac{A_{d}}{\left|s_{\|}\right|^{2 \hat{\Delta}}} \delta_{\hat{O}_{1}^{i} \hat{O}_{2}^{j}}
$$

The e.o.m. is found by varying the action w.r.t. $\phi$, where we assume the bulk to have a sextic interaction near three dimensions and a quartic near four dimensions $[29,31]$

$$
\partial^{2} \phi^{i}=\lambda_{n} \phi^{a_{n}-2} \phi^{i}, \quad \lambda_{n}=\frac{8 \pi^{2} \epsilon}{3 N+22} \delta_{n, 3}+\frac{8 \pi^{2} \epsilon}{N+8} \delta_{n, 4}, \quad a_{n}=6 \delta_{n, 3}+4 \delta_{n, 4} .
$$

Here $n$ denotes the integer part of the spacetime dimension, $\lambda_{n}$ is the WF coupling constant at the RG fixed point and $\phi^{a_{n}-2}$ is a composite operator. By dimensional analysis of the corresponding Lagrangian, $\Delta_{\phi}^{(\text {free })}$ is given by (3.9). If we let the derivatives on the l.h.s. of the e.o.m. act on the correlator (3.27)

$$
\begin{aligned}
\text { L.h.s. } & =\left\langle\partial^{2} \phi^{i}(x) \phi^{j}(y)\right\rangle=\partial_{x_{\|}}^{2}\left\langle\phi^{i}(x) \phi^{j}(y)\right\rangle+\partial_{z}^{2}\left\langle\phi^{i}(x) \phi^{j}(y)\right\rangle \\
& =\delta^{i j} \mu_{\hat{\Delta}} \sum_{m \geq 0} \frac{a_{\hat{\Delta}, m-1}+c_{\hat{\Delta}, m} a_{\hat{\Delta}, m}}{|z|^{\Delta_{\phi}}-\hat{\Delta}-2 m+2} \partial_{\|}^{2 m} \frac{1}{\left|s_{\|}\right|^{2 \hat{\Delta}}}, \\
c_{\hat{\Delta}, m} & =\left(\Delta_{\phi}-\hat{\Delta}-2 m\right)\left(\Delta_{\phi}-\hat{\Delta}-2 m+1\right) .
\end{aligned}
$$

The summand can be simplified to

$$
a_{\hat{\Delta}, m-1}+c_{\hat{\Delta}, m} a_{\hat{\Delta}, m}=\frac{\left(\Delta_{\phi}-\hat{\Delta}\right)^{2}+\left(\Delta_{\phi}-\hat{\Delta}\right)+2 m\left[d-2\left(\Delta_{\phi}+1\right)\right]}{(-4)^{m} m !} \frac{\Gamma_{\hat{\Delta}-(d-3) / 2}}{\Gamma_{m+\hat{\Delta}-(d-3) / 2}} .
$$

We can choose a renormalization scheme such that the one-point function of $\left\langle\phi^{2}\right\rangle$ is the finite part of the coincident limit of $\langle\phi(x) \phi(y)\rangle$. We assume it to be given by

$$
\left\langle\phi^{2}(x)\right\rangle=\frac{\kappa_{n}^{ \pm}}{|z|^{2 \Delta_{\phi}}}+\mathcal{O}\left(\lambda_{n}\right) .
$$

The coefficient $\kappa_{n}^{ \pm}$can be found in table 1 for different models. The r.h.s. of the e.o.m. (3.29) acting on the correlator (3.27) is found using Wick's theorem

$$
\begin{aligned}
\text { R.h.s. } & =\left\langle\lambda_{n} \phi^{a_{n}-2} \phi^{i}(x) \phi^{j}(y)\right\rangle=\delta^{i j} \lambda_{n} \sigma_{n}\left\langle\phi^{2}(x)\right\rangle^{a_{n}-2}\langle\phi(x) \phi(y)\rangle \\
& =\delta^{i j} \mu_{\hat{\Delta}} \sum_{m \geq 0}\left(a_{\hat{\Delta}, m} \frac{\lambda_{n} \sigma_{n}\left(\kappa_{n}^{ \pm}\right)^{a_{n}-2}}{|z|^{\Delta_{\phi}-\hat{\Delta}-2 m+2}} \partial_{\|}^{2 m} \frac{A_{d}}{\left|s_{\|}\right|^{2 \hat{\Delta}}}+\mathcal{O}\left(\lambda_{n}^{2}, \lambda_{n} \epsilon\right)\right), \\
\sigma_{n} & =(N+4)(N+2) \delta_{n, 3}+(N+2) \delta_{n, 4} .
\end{aligned}
$$




\begin{tabular}{|l|l|l|l|}
\hline Type of defect: & $n$ & $\kappa_{n}^{ \pm}$ & $\left(\gamma_{n}^{ \pm}\right)^{(1)}$ \\
\hline Boundary & 3 & $\left( \pm \frac{A_{3}}{2}\right)^{2}=\frac{1}{64 \pi^{2}}$ & $\mp \frac{(N+4)(N+2)}{8(3 N+22)}$ \\
\hline Boundary & 4 & $\pm \frac{A_{4}}{2}= \pm \frac{1}{16 \pi^{2}}$ & $-\frac{N+2}{2(N+8)}$ \\
\hline Interface & 4 & 0 & 0 \\
\hline
\end{tabular}

Table 1. The table shows the one-point functions of $\phi^{2}$ and anomalous dimensions in different models: $n$ denotes the integer part of the spacetime dimension, and the subscript " \pm " only applies to BCFTs where "+" means Neumann b.c., and "-" means Dirichlet.

Here $\sigma_{n}$ is the symmetry factor from Wick's theorem. We can compare this to the l.h.s. (3.30) to find an equation for the scaling dimensions that holds for all $m \in \mathbb{Z}_{\geq 0}$ up to order $\epsilon$

$$
a_{\hat{\Delta}, m-1}+c_{\hat{\Delta}, m} a_{\hat{\Delta}, m}=a_{\hat{\Delta}, m} \lambda_{n} \sigma_{n}\left(\kappa_{n}^{ \pm}\right)^{a_{n}-2} .
$$

Let us first solve this equation for the free theory when the l.h.s. is zero on its own. Then either the $\Gamma$-functions are zero or the numerator is zero in equation (3.31). The $\Gamma$-functions are zero when $\hat{\Delta}=\Delta_{\phi}^{(\text {free })}-(m+k), m+k \geq 1, m, k \in \mathbb{Z}_{\geq 0}$. However, these solutions are not valid if the CFT on the boundary is unitary.

The numerator on the other hand is zero when

$$
\hat{\Delta}_{+}^{(\text {free })}=\Delta_{\phi}^{(\text {free })}, \quad \text { or, } \quad \hat{\Delta}_{-}^{(\text {free })}=\Delta_{\phi}^{(\text {free })}+1 .
$$

These are the scaling dimensions of $\hat{\phi}$ and $\partial_{\perp} \hat{\phi}$. In a BCFT they correspond to Neumann or Dirichlet b.c.'s respectively. This proves that these are the only operators that can appear in the BOE of $\phi$ in the free theory. Note that this result is independent of the spacetime dimension and also applies to ICFTs. This result is not new in itself, and has been found prior to this work in $[1,18]$.

In the interacting theory, we expand the scaling dimensions in $\epsilon$, using as input from the bulk theory that the fundamental scalar in the bulk has no anomalous dimension at this order

$$
\begin{aligned}
& \Delta_{\phi}=\Delta_{\phi}^{\text {(free) }}+\mathcal{O}\left(\epsilon^{2}\right), \\
& \hat{\Delta}_{ \pm}=\hat{\Delta}_{ \pm}^{\text {(free) }}+\epsilon\left(\gamma_{n}^{ \pm}\right)^{(1)}+\mathcal{O}\left(\epsilon^{2}\right) .
\end{aligned}
$$

The e.o.m. (3.34) has one solution that holds for any $m$

$$
\left(\gamma_{n}^{ \pm}\right)^{(1)}=\mp \frac{\lambda_{n} \sigma_{n}\left(\kappa_{n}^{ \pm}\right)^{a_{n}-2}}{\epsilon} .
$$

We list the anomalous dimensions for different models in table 1. The BCFT results near three and four dimensions match with the existing literature [32-36].

Of particular interest for us, the anomalous dimensions of $\hat{\phi}$ and $\partial_{\perp} \hat{\phi}$ in the ICFT that was considered in the previous section are both zero. ${ }^{12}$ Thus from (3.14) we can deduce that

$$
\alpha=0 \text {. }
$$

This is the last constraint we find on the CFT data in the ICFT we are considering.

\footnotetext{
${ }^{12}$ One can also see this by letting the e.o.m. act on the resummed correlator at order $\epsilon$.
} 
Let us remind the reader that we consider another interface than those studied in $[22-$ $26]$, where the anomalous dimensions of $\hat{\phi}$ and $\partial_{\perp} \hat{\phi}$ are not zero.

\subsection{CFT data at order $\epsilon^{2}$}

In this subsection we summarize the CFT data related to the interface

$$
\begin{aligned}
\lambda a_{0} & =\epsilon \beta+\epsilon^{2} \frac{2 \gamma_{\phi}^{(2)}-\hat{\gamma}_{0}^{(2)}-\hat{\gamma}_{1}^{(2)}}{2}+\mathcal{O}\left(\epsilon^{3}\right), \\
\lambda a_{1} & =-\epsilon^{2} \frac{2 \beta \gamma_{0}^{(1)}-2 \gamma_{\phi}^{(2)}+3 \hat{\gamma}_{0}^{(2)}-\hat{\gamma}_{1}^{(2)}}{4}+\mathcal{O}\left(\epsilon^{3}\right), \\
\lambda a_{n \geq 2} & =\epsilon^{2} \frac{(-1)^{n}(n !)^{2}}{(2 n) !} \frac{\beta \gamma_{0}^{(1)}+\hat{\gamma}_{0}^{(2)}-\hat{\gamma}_{1}^{(2)}}{n}+\mathcal{O}\left(\epsilon^{3}\right), \\
\mu_{0}^{2} & =1+\mathcal{O}\left(\epsilon^{3}\right), \\
\mu_{1}^{2} & =\frac{\Delta_{\phi}^{(\text {free })}}{2}+\epsilon^{2} \frac{\beta\left(1-2 \gamma_{0}^{(1)}\right)+4 \gamma_{\phi}^{(2)}-2 \hat{\gamma}_{0}^{(2)}}{4}+\mathcal{O}\left(\epsilon^{3}\right), \\
\mu_{m \geq 2}^{2} & =\epsilon^{2} \frac{(m-2) !}{(3 / 2)_{m-2}} \frac{2 \gamma_{\phi}^{(2)}+(-1)^{m}\left(\beta \gamma_{0}^{(1)}+\hat{\gamma}_{0}^{(2)}-\hat{\gamma}_{1}^{(2)}\right)}{4^{m-1}}+\mathcal{O}\left(\epsilon^{3}\right) .
\end{aligned}
$$

The full correlator expressed in the anomalous dimensions is given by

$$
\begin{aligned}
F_{ \pm \pm}(\xi)= & +\epsilon \beta \frac{\xi}{\xi+1}-\frac{\epsilon^{2}}{2}\left(\beta\left(1-\gamma_{0}^{(1)}\right) \frac{\xi}{\xi+1} \log (\xi)+\right. \\
& \left.-\left(\left(2 \gamma_{\phi}^{(2)}-\hat{\gamma}_{0}^{(2)}-\hat{\gamma}_{1}^{(2)}\right)+\left(\beta\left(1-\gamma_{0}^{(1)}\right)-\hat{\gamma}_{0}^{(2)}+\hat{\gamma}_{1}^{(2)}\right) \frac{\xi}{\xi+1}\right) \log (\xi+1)\right)+\mathcal{O}\left(\epsilon^{3}\right) .
\end{aligned}
$$

We can resum the correlator in $\epsilon$ to find

$$
\left\langle\phi_{ \pm}^{i}(x) \phi_{ \pm}^{j}(y)\right\rangle=\delta^{i j}\left(\frac{(\xi+1)^{\gamma_{\phi}-\left(\hat{\gamma}_{0}+\hat{\gamma}_{1}\right) / 2}}{|x-y|^{2 \Delta_{\phi}}}+\epsilon \beta \frac{(\xi+1)^{\left(\hat{\gamma}_{1}-\hat{\gamma}_{0}\right) / 2 \beta}}{|\tilde{x}-y|^{2 \Delta_{\phi}}}\left(\frac{\xi}{\xi+1}\right)^{\gamma_{0} / 2-\gamma_{\phi}}\right)+\mathcal{O}\left(\epsilon^{3}\right) .
$$

Here $\Delta_{\phi}$ is the full scaling dimension of $\phi$ (with the anomalous dimensions included), $\gamma_{\phi}, \gamma_{0}, \hat{\gamma}_{0}$ as well as $\hat{\gamma}_{1}$ are the full anomalous dimensions (to all orders of $\epsilon$ ), and $\tilde{x}=\left.x\right|_{z \rightarrow-z}$ is the image point of $x$. Note that this correlator does not satisfy the image symmetry $\left(z, z^{\prime}\right) \rightarrow\left(-z,-z^{\prime}\right)$. To our knowledge the anomalous dimensions of the interface fields are not known. The anomalous dimensions of $\phi$ and $\phi^{2}$ are known [29] and can be used as input from the bulk theory

$$
\gamma_{0}=\frac{N+2}{N+8} \epsilon+\mathcal{O}\left(\epsilon^{2}\right), \quad \gamma_{\phi}=\frac{N+2}{4(N+8)^{2}} \epsilon^{2}+\mathcal{O}\left(\epsilon^{3}\right) .
$$

\subsection{The renormalization group domain wall}

Let us now specialize to the case when one side of the interface is free, say the side with $\phi_{-}$at $z<0$. This model was studied in [1]. Since the interface fields are limits of the free fields in the bulk at $z<0$ (as seen from the b.c.'s (3.1)), they are all protected. This 
means that we can retrieve the full $\phi_{+}-\phi_{+}$correlator as well as the OPE coefficients from (3.39), (3.40) and (3.41) by setting the interface anomalous dimensions to zero ${ }^{13}$

$$
\hat{\gamma}_{m}=0 \forall m \in \mathbb{Z}_{\geq 0}
$$

This yields the correlator

$$
\left\langle\phi_{+}^{i}(x) \phi_{+}^{j}(y)\right\rangle=\delta^{i j}\left(\frac{(\xi+1)^{\gamma_{\phi}}}{|x-y|^{2 \Delta_{\phi}}}+\frac{\epsilon \beta}{|\tilde{x}-y|^{2 \Delta_{\phi}}}\left(\frac{\xi}{\xi+1}\right)^{\gamma_{0} / 2-\gamma_{\phi}}\right)+\mathcal{O}\left(\epsilon^{3}\right) .
$$

We find $\left\langle\phi_{-}^{i} \phi_{-}^{j}\right\rangle$ from $\left\langle\phi_{+}^{i} \phi_{+}^{j}\right\rangle$ by setting the bulk anomalous dimensions to zero

$$
\gamma_{\phi}=0 \Rightarrow\left\langle\phi_{-}^{i} \phi_{-}^{j}\right\rangle=\delta^{i j}\left(\frac{1}{|x-y|^{2 \Delta_{\phi}^{(\text {free }}}}+\frac{\epsilon \beta}{|\tilde{x}-y|^{2 \Delta_{\phi}^{(\text {free })}}}\right)+\mathcal{O}\left(\epsilon^{3}\right) .
$$

Note that even though both the bulk and the boundary operators are protected, this correlator still differs from that in a homogeneous CFT.

\section{A boundary CFT in $6-\epsilon$ dimensions}

In this section we study the $\phi^{3}$-theory in $d=6-\epsilon$ dimensions close to a boundary. The bulk theory is called the Landau-Ginzburg model and it describes the distribution of "Yang-Lee zeros" (the zeros of the partition function) on the imaginary magnetic field axis in ferromagnets above the critical temperature [37]. The model is not unitary so the OPE coefficients do not need to be real-valued.

This model was considered in the presence of a boundary [38], and a generalization of it with a tensorial coupling constant and $N$-vector fields was studied in $[39,40]$. It describes the continuum limit of the $(N+1)$-state Potts model, where $N \rightarrow 0$ corresponds to percolation, and $N=1$ describes an extended Ising model. The case of $N=1$ belongs to another universality class than $N \geq 2$, and thus our result may differ from those in [39, 40].

We consider the two-point function (2.1) of the fundamental scalar $\phi$ of dimension $\Delta_{\phi}$, and bootstrap this correlator upto $\mathcal{O}(\epsilon)$. This two-point function in the free theory reads

$$
F^{ \pm}(\xi)=1 \pm\left(\frac{\xi}{\xi+1}\right)^{\Delta_{\phi}^{(\text {free })}}, \quad \Delta_{\phi}^{(\text {free })}=\frac{d-2}{2} .
$$

Here "+" and "_" represent Neumann and Dirichlet b.c.'s respectively

$$
\begin{aligned}
\partial_{\perp} \hat{\phi} & =0 \\
\hat{\phi} & =0 .
\end{aligned}
$$

The $\phi^{2}$-operator is a descendant of $\phi$ in the interacting theory, which can be seen from the e.o.m. $\partial^{2} \phi \sim \phi^{2}$. This means that the operator $\phi$ appears in the bulk OPE of $\phi \times \phi$ instead of $\phi^{2}$ (this is a consequence of breaking $\mathbb{Z}_{2}$-symmetry). The bulk conformal

\footnotetext{
${ }^{13}$ We checked this result using the same bootstrap procedure as in section 3.1.
} 
block corresponding to the exchange of $\phi$ contains a simple pole in $\epsilon$. This singularity is compensated by including the anomalous dimension of $\phi$ already in the free theory. The decomposition is

$$
\begin{aligned}
\lambda a_{\mathbb{1}} & =1, \quad \lambda a_{\Delta}^{ \pm}=\mp \epsilon \gamma_{\phi}^{(1)} \delta_{\Delta, \Delta_{\phi}}, \\
\mu_{\mathbb{1}}^{2} & =0, \quad\left(\mu_{\hat{\Delta}}^{ \pm}\right)^{2}=(1 \pm 1) \delta_{\hat{\Delta}, \Delta_{\phi}^{(\text {free })}}+(1 \mp 1) \frac{\Delta_{\phi}^{(\text {free })}}{2} \delta_{\hat{\Delta}, \Delta_{\phi}^{(\text {free })}+1},
\end{aligned}
$$

where $\gamma_{\phi}^{(1)}$ is the anomalous dimension of $\phi$ at order $\epsilon$. This block decomposition indicates that only the bulk identity and $\phi$ contribute in the bulk-channel whereas only $\hat{\phi}$ or $\partial_{\perp} \hat{\phi}$ contributes in the boundary-channel with Neumann or Dirichlet b.c. respectively. This agrees with the results from section 3.2 even though this theory is non-unitary.

We will now use the bootstrap equation (2.3) to constrain the CFT data at higher orders in $\epsilon$. The CFT data admits an expansion in $\sqrt{\epsilon}$. One can check (either by expanding in $\xi$, or by using the method presented in this paper), that all of the CFT data in the bootstrap equation is zero at order $\sqrt{\epsilon}$. We will thus expand the CFT data as

$$
\begin{aligned}
\Delta_{\phi} & =\Delta_{\phi}^{(\text {free })}+\epsilon \gamma_{\phi}^{(1)}+\mathcal{O}\left(\epsilon^{3 / 2}\right) \\
\Delta_{n \geq 1} & =2\left(\Delta_{\phi}^{(\text {free })}+n\right)+\epsilon \gamma_{n}^{(1)}+\mathcal{O}\left(\epsilon^{3 / 2}\right) \\
\hat{\Delta}_{m \geq 2} \equiv \Delta_{\partial_{\perp}^{m} \hat{\phi}} & =\Delta_{\phi}^{(\text {free })}+m+\epsilon \hat{\gamma}_{m}^{(1)}+\mathcal{O}\left(\epsilon^{3 / 2}\right) \\
\lambda a_{\mathbb{1}} & =1 \\
\lambda a_{\phi}^{ \pm} & =\mp \epsilon \gamma_{\phi} \delta_{\Delta, \Delta_{\phi}}+\epsilon^{2}\left(\lambda a_{\phi}^{(2)}\right)^{ \pm}+\mathcal{O}\left(\epsilon^{5 / 2}\right), \\
\lambda a_{\Delta_{n}}^{ \pm} & =\epsilon\left(\lambda a_{n}^{(1)}\right)^{ \pm}+\mathcal{O}\left(\epsilon^{3 / 2}\right) \\
\mu_{\mathbb{1}}^{2} & =0 \\
\mu_{\hat{\Delta}_{m}}^{2} & =(1 \pm 1) \delta_{m, 0}+(1 \mp 1) \frac{\Delta_{\phi}^{(\text {free })}}{2} \delta_{m, 1}+\epsilon\left(\mu_{m}^{(1)}\right)^{ \pm}+\mathcal{O}\left(\epsilon^{3 / 2}\right) .
\end{aligned}
$$

Let us write the bootstrap equation at order $\epsilon$ as

$$
G_{b}^{ \pm}(\xi)+H_{b}^{ \pm}(\xi)+\mathcal{O}\left(\epsilon^{3 / 2}\right)=G_{i}^{ \pm}(\xi)+H_{i}^{ \pm}(\xi)+\mathcal{O}\left(\epsilon^{3 / 2}\right),
$$

where $H_{b}$ and $H_{i}$ correspond to new operators

$$
\begin{aligned}
& G_{b}^{ \pm}(\xi)=\left(\mp \epsilon \gamma_{\phi}^{(1)} \delta_{\Delta, \Delta_{\phi}}+\epsilon^{2}\left(\lambda a_{\phi}^{(2)}\right)^{ \pm}\right) \mathcal{G}_{\mathrm{ope}}\left(\Delta_{\phi} ; \xi\right) \\
& G_{i}^{ \pm}(\xi)=(1 \pm 1) \mathcal{G}_{\mathrm{boe}}\left(\hat{\Delta}_{0} ; \xi\right)+(1 \mp 1) \frac{\Delta_{\phi}^{(\text {free })}}{2} \mathcal{G}_{\text {boe }}\left(\hat{\Delta}_{1} ; \xi\right) \\
& H_{b}^{ \pm}(\xi)=\epsilon \sum_{n \geq 1}\left(\lambda a_{n}^{(1)}\right)^{ \pm} \mathcal{G}_{\mathrm{ope}}(2(n+1) ; \xi) \\
& H_{i}^{ \pm}(\xi)=\epsilon \sum_{m \geq 0}\left(\mu_{m}^{(1)}\right)^{ \pm} \mathcal{G}_{\mathrm{boe}}(m+1 ; \xi) .
\end{aligned}
$$

We included the bulk $\phi$-exchange in $G_{b}$ since its conformal block does not have a branch cut along $\xi<-1$. 
Now take the discontinuity (2.6) around $\xi<-1$, and assume that it commutes with the series' in $H_{b}$ and $H_{i}$ (2.17). We proceed to project out the bulk OPE coefficients (2.18) using the orthogonality relation (2.8) of the Jacobi polynomials

$$
\begin{aligned}
\left(\lambda a_{n}^{(1)}\right)^{ \pm} & =\frac{(-1)^{n}(n-1) !(n+1) !}{(2 n) !} \int_{-1}^{+1} d y P_{n-1}^{(2,0)}(y)\left(A_{1}^{ \pm}+A_{2}^{ \pm}(1-y)^{2}\right) \\
A_{1}^{ \pm} & =\frac{ \pm\left(1-2 \gamma_{\phi}^{(1)}\right)+2(1 \pm 1) \hat{\gamma}_{0}^{(1)}-2(1 \mp 1) \hat{\gamma}_{1}^{(1)}}{8} \\
A_{2}^{ \pm} & =-\frac{2 \gamma_{\phi}^{(1)}-(1 \pm 1) \hat{\gamma}_{0}^{(1)}-(1 \mp 1) \hat{\gamma}_{1}^{(1)}}{16} .
\end{aligned}
$$

Performing the integral over $y$ yields

$$
\left(\lambda a_{1}^{(1)}\right)^{ \pm}=-2 A_{1}^{ \pm}-\frac{8}{3} A_{2}^{ \pm}, \quad\left(\lambda a_{n \geq 2}^{(1)}\right)^{ \pm}=2 \frac{(-1)^{n}(n-1) !(n+1) !}{(2 n) !} A_{1}^{ \pm} .
$$

The bulk-channel is resummed using the integral representation (B.1)

$$
H_{b}^{ \pm}=-\epsilon\left(\frac{\xi}{\xi+1}\left(6 A_{1}^{ \pm}+\left(5 A_{1}^{ \pm}+\frac{8}{3} A_{2}^{ \pm}\right) \frac{\xi}{\xi+1}\right)-\left(6 A_{1}^{ \pm}-2 A_{2}^{ \pm}\left(\frac{\xi}{\xi+1}\right)^{2}\right) \log (\xi+1)\right) .
$$

With this we can find the full correlator. If we impose Neumann or Dirichlet b.c.'s we also get rid of unwanted branch cuts along $\xi \in(-1,0)$ in $H_{i}^{ \pm}$from the bootstrap equation $(4.8)^{14}$

$$
\begin{array}{llrl}
\left(\lambda a_{\phi}^{(2)}\right)^{+} & =-\frac{17}{32} \gamma_{\phi}^{(1)}\left(1+2 \gamma_{\phi}^{(1)}\right), & \hat{\gamma}_{0}^{(1)}=-\frac{3}{16}+\frac{5}{8} \gamma_{\phi}^{(1)}, \\
\left(\lambda a_{\phi}^{(2)}\right)^{-}=-\frac{\gamma_{\phi}^{(1)}\left(1+2 \gamma_{\phi}^{(1)}\right)}{16}, & \hat{\gamma}_{1}^{(1)}=-\frac{3}{2}+\frac{\gamma_{\phi}^{(1)}}{4} .
\end{array}
$$

We project out the BOE coefficients from $H_{i}^{ \pm}$using the orthogonality relation (2.9)

$$
\begin{aligned}
& \left(\mu_{m}^{(1)}\right)^{ \pm}=\oint_{|w|=\tilde{\epsilon}} \frac{d w}{2 \pi i} \frac{{ }_{2} F_{1}(-1-m, 1-m, 2(1-m),-w)}{w^{m+1}}\left(B_{1}^{ \pm}+B_{2}^{ \pm} \frac{w}{w+1}+\right. \\
& \left.+B_{3}^{ \pm}\left(\frac{w}{w+1}\right)^{2}+\left(B_{4}^{ \pm}+B_{5}^{ \pm} \frac{w}{w+1}+B_{6}^{ \pm}\left(\frac{w}{w+1}\right)^{2}\right) \log (w+1)\right) . \\
& B_{1}^{+}=-\frac{1}{4}-\frac{\gamma_{\phi}^{(1)}}{2}, \quad B_{2}^{+}=\frac{5}{16}-\frac{3}{8} \gamma_{\phi}^{(1)}, \quad B_{3}^{+}=-\frac{1}{4}+\frac{3}{2} \gamma_{\phi}^{(1)}, \\
& B_{4}^{+}=-\frac{1}{16}+\frac{7}{8} \gamma_{\phi}^{(1)}, \quad B_{5}^{+}=\frac{1}{8}+\frac{\gamma_{\phi}^{(1)}}{4}, \quad B_{6}^{+}=-\frac{1}{16}-\frac{\gamma_{\phi}^{(1)}}{8} \text {, } \\
& B_{1}^{-}=0, \quad B_{2}^{-}=0, \quad B_{3}^{-}=\frac{3}{16}-\frac{\gamma_{\phi}^{(1)}}{8}, \\
& B_{4}^{-}=-\frac{1}{8}+\frac{3}{4} \gamma_{\phi}^{(1)}, \quad B_{5}^{-}=\frac{1}{4}+\frac{\gamma_{\phi}^{(1)}}{2}, \quad B_{6}^{-}=-\frac{1}{8}-\frac{\gamma_{\phi}^{(1)}}{4},
\end{aligned}
$$

\footnotetext{
${ }^{14}$ For Neumann b.c.'s, there is a simple pole in $z$ in the boundary limit of $\partial_{\perp} \phi$, which corresponds to the expected exchange of $\hat{\phi}$ in the BOE. This term do not need to be zero. Only the $z^{0}$-term corresponding to the exchange of $\partial_{\perp} \hat{\phi}$ should be set to zero.
} 
Calculating the residues gives us the BOE coefficients

$$
\begin{aligned}
&\left(\mu_{0}^{(1)}\right)^{ \pm}=B_{1}^{ \pm}, \\
&\left(\mu_{1}^{(1)}\right)^{-}=B_{2}^{-}+B_{4}^{-}, \\
&\left(\mu_{2}^{(1)}\right)^{ \pm}=\frac{B_{2}^{ \pm}}{2}+B_{3}^{ \pm}+B_{4}^{ \pm}+B_{5}^{ \pm}, \\
&\left(\mu_{m \geq 3}^{(1)}\right)^{ \pm}= \frac{\sqrt{\pi} \Gamma_{m-2}}{\Gamma_{m-1 / 2}} \\
& \times \frac{(m-2)(m+1) B_{4}^{ \pm}+\left(m(m-1)-1-(-1)^{m}\right) B_{5}^{ \pm}+m(m-1)\left(1-(-1)^{m}\right) B_{6}^{ \pm}}{4^{m-1}} .
\end{aligned}
$$

We have to be a bit careful as $\partial_{\perp} \hat{\phi}$ (corresponding to $\mu_{1}^{+}$) does not appear for Neumann b.c.'s (even though its coefficient seems to be non-zero from the calculations). ${ }^{15}$ One can see that this block should not appear by resumming the boundary-channel, ${ }^{16}$ and comparing the result with the $H_{i}^{+}$found from the bootstrap equation (4.8). While resumming the boundary-channel we find that the property (2.11) is trivially satisfied for both even and odd operators.

The BOE coefficients above, together with the bulk OPE coefficients and boundary anomalous dimensions at (4.11) and (4.12) are the constraints on the CFT data found from the bootstrap equation. They are all expressed in terms of one parameter: the anomalous dimension of the external field $\phi$. We were not able to implement the e.o.m. in such a way that we could find this parameter. Hence we will use it as an input $[41,42]^{17}$

$$
\gamma_{\phi}^{(1)}=-\frac{1}{18} \quad \Rightarrow \quad\left(\lambda a_{1}^{(1)}\right)^{ \pm}=0
$$

The non-trivial CFT data for Neumann b.c.'s is given by

$$
\begin{aligned}
\hat{\gamma}_{0} & =-\frac{2}{9} \epsilon+\mathcal{O}\left(\epsilon^{3 / 2}\right), \\
\lambda a_{\phi}^{+} & =\frac{\epsilon}{18}+\frac{17}{648} \epsilon^{2}+\mathcal{O}\left(\epsilon^{5 / 2}\right), \\
\lambda a_{n \geq 2}^{+} & =\frac{(-1)^{n}(n-1) !(n+1) !}{18(2 n) !} \epsilon+\mathcal{O}\left(\epsilon^{3 / 2}\right), \\
\left(\mu_{0}^{+}\right)^{2} & =2-\frac{2}{9} \epsilon+\mathcal{O}\left(\epsilon^{3 / 2}\right), \\
\left(\mu_{2}^{+}\right)^{2} & =-\frac{\epsilon}{6}+\mathcal{O}\left(\epsilon^{3 / 2}\right), \\
\left(\mu_{m \geq 3}^{+}\right)^{2} & =-\frac{\sqrt{\pi}(m+1) \Gamma_{m-1}\left(1-(-1)^{m}\right)}{2^{2 m-1} \Gamma_{m-1 / 2}} \frac{\epsilon}{9}+\mathcal{O}\left(\epsilon^{3 / 2}\right),
\end{aligned}
$$

\footnotetext{
${ }^{15}$ Physically the normal derivative is forbidden due to the Neumann b.c. (4.2).

${ }^{16}$ We resum even and odd parts separately using the procedure in appendix C.2.

${ }^{17}$ Note that these references consider $d=6-2 \epsilon$ while we consider $d=6-\epsilon$.
} 
where $\hat{\gamma}_{0}$ agrees with the result from [38], and the non-trivial CFT data for Dirichlet b.c.'s is given by

$$
\begin{aligned}
\hat{\gamma}_{1} & =-\frac{7}{18} \epsilon+\mathcal{O}\left(\epsilon^{3 / 2}\right) \\
\lambda a_{\phi}^{-} & =-\frac{\epsilon}{18}+\frac{\epsilon^{2}}{324}+\mathcal{O}\left(\epsilon^{5 / 2}\right) \\
\lambda a_{n \geq 2}^{-} & =\frac{(-1)^{n}(n-1) !(n+1) !}{9(2 n) !} \epsilon+\mathcal{O}\left(\epsilon^{3 / 2}\right) \\
\left(\mu_{1}^{-}\right)^{2} & =\Delta_{\phi}^{(\text {free })}-\frac{\epsilon}{6}+\mathcal{O}\left(\epsilon^{3 / 2}\right) \\
\left(\mu_{2}^{-}\right)^{2} & =\frac{\epsilon}{4}+\mathcal{O}\left(\epsilon^{3 / 2}\right) \\
\left(\mu_{m \geq 3}^{-}\right)^{2} & =-\frac{\sqrt{\pi}(m+1) \Gamma_{m-1}\left(1-2(-1)^{m}\right)}{2^{2 m-1} \Gamma_{m-1 / 2}} \frac{\epsilon}{9}+\mathcal{O}\left(\epsilon^{3 / 2}\right) .
\end{aligned}
$$

The full correlators reads

$$
\begin{aligned}
F^{+}(\xi)=1 & +\left(\frac{\xi}{\xi+1}\right)^{2}-\frac{\epsilon}{3}\left(\frac{1}{3}\left(1+\frac{\xi}{\xi+1}\right) \frac{\xi}{\xi+1}+\frac{5}{6}\left(\frac{\xi}{\xi+1}\right)^{2} \log (\xi)+\right. \\
& \left.-\left(\frac{1}{2}+\frac{4}{3}\left(\frac{\xi}{\xi+1}\right)^{2}\right) \log (\xi+1)\right)+\mathcal{O}\left(\epsilon^{3 / 2}\right) \\
F^{-}(\xi)= & -\left(\frac{\xi}{\xi+1}\right)^{2}-\frac{\epsilon}{3}\left(\frac{7}{6}\left(1-\frac{\xi}{\xi+1}\right) \frac{\xi}{\xi+1}-\frac{5}{6}\left(\frac{\xi}{\xi+1}\right)^{2} \log (\xi)+\right. \\
& \left.-\left(1-\frac{11}{6}\left(\frac{\xi}{\xi+1}\right)^{2}\right) \log (\xi+1)\right)+\mathcal{O}\left(\epsilon^{3 / 2}\right)
\end{aligned}
$$

\section{An interface CFT in $6-\epsilon$ dimensions}

In this section we bootstrap an ICFT in $6-\epsilon$ dimensions with cubic interactions on both sides of it. We will consider the same b.c.'s (3.1) as in section 3 (without the $O(N)$-indices), giving us the free theory correlator (3.7) and the free theory decomposition (3.9) and (3.10).

We can proceed to bootstrap this correlator at order $\sqrt{\epsilon}$, assuming that the bulk anomalous dimensions does not receive corrections at this order ${ }^{18}$

$$
\begin{aligned}
\Delta_{\phi} & =\Delta_{\phi}^{(\text {free })}+\epsilon \gamma_{\phi}^{(1)}+\mathcal{O}\left(\epsilon^{3 / 2}\right), \\
\Delta_{n \geq 1} & =2\left(\Delta_{\phi}^{(\text {free })}+n\right)+\mathcal{O}(\epsilon), \\
\hat{\Delta}_{m \geq 2} \equiv \Delta_{\partial_{\perp}^{m} \hat{\phi}} & =\Delta_{\phi}^{(\text {free })}+m+\sqrt{\epsilon} \hat{\gamma}_{m}^{(1 / 2)}+\mathcal{O}(\epsilon), \\
\lambda a_{\mathbb{1}} & =1, \\
\lambda a_{\phi} & =\epsilon^{3 / 2} \lambda a_{\phi}^{(3 / 2)}+\mathcal{O}\left(\epsilon^{2}\right), \\
\lambda a_{\Delta_{n}} & =\sqrt{\epsilon} \lambda a_{n}^{(1 / 2)}+\mathcal{O}(\epsilon),
\end{aligned}
$$

\footnotetext{
${ }^{18}$ This assumption is motivated by the trivial CFT data one finds at order $\sqrt{\epsilon}$ in the BCFT case.
} 


$$
\begin{aligned}
\mu_{\mathbb{1}}^{2} & =0, \\
\mu_{\hat{\Delta}_{m}}^{2} & =\delta_{m, 0}+\frac{\Delta_{\phi}^{(\text {free })}}{2} \delta_{m, 1}+\sqrt{\epsilon} \mu_{m}^{(1 / 2)}+\mathcal{O}(\epsilon) .
\end{aligned}
$$

Note that due to the pole in the $\phi$-block, one has to consider higher orders in its corresponding CFT data. The bootstrap calculations are very similar to those in section $4 .{ }^{19}$ We are able to express all of the CFT data in terms of one interface anomalous dimension

$$
\begin{aligned}
\hat{\gamma}_{1}^{(1 / 2)} & =2 \hat{\gamma}_{0}^{(1 / 2)} \\
\lambda a_{\phi}^{(3 / 2)} & =\frac{9}{4} \gamma_{\phi}^{(1)} \hat{\gamma}_{0}^{(1 / 2)}=-\frac{\hat{\gamma}_{0}^{(1 / 2)}}{8}, \\
\lambda a_{1}^{(1 / 2)} & =0 \\
\lambda a_{n \geq 2}^{(1 / 2)} & =-\frac{(-1)^{n}(n-1) !(n+1) !}{(2 n) !} \frac{\hat{\gamma}_{0}^{(1 / 2)}}{2}, \\
\mu_{0}^{(1 / 2)} & =0 \\
\mu_{1}^{(1 / 2)} & =\hat{\gamma}_{0}^{(1 / 2)}, \\
\mu_{2}^{(1 / 2)} & =-\frac{3 \hat{\gamma}_{0}^{(1 / 2)}}{4}, \\
\mu_{m \geq 3}^{(1 / 2)} & =-\frac{\sqrt{\pi}(-1)^{m}(m+1) \Gamma_{m-1}}{2^{2 m-1} \Gamma_{m-1 / 2}} \hat{\gamma}_{0}^{(1 / 2)} .
\end{aligned}
$$

The constraints on $\hat{\gamma}_{1}^{(1 / 2)}$ and $\lambda a_{\phi}^{(3 / 2)}$ are found by removing branch cuts in $H_{i}$ as well as resumming the boundary-channel and comparing it with the original $H_{i}$ respectively. The full correlator is given by

$$
F(\xi)=1+\frac{\sqrt{\epsilon} \hat{\gamma}_{0}^{(1 / 2)}}{2}\left(3 \frac{\xi}{\xi+1}\left(1-\frac{\xi}{\xi+1}\right)-\left(3-\left(\frac{\xi}{\xi+1}\right)^{2}\right) \log (\xi+1)\right)+\mathcal{O}(\epsilon) .
$$

For the RG domain wall near six dimensions (when one side of the interface is free), there are no corrections at order $\sqrt{\epsilon}$. In principle one could proceed to bootstrap at order $\epsilon$, although we are only able to commute the discontinuities with the series' in the boundarychannel in the case when the theory is completely free. It seems like more delicate methods are required in order to bootstrap this theory at order $\epsilon{ }^{20}$

\section{Conclusions}

In this paper we studied a CFT with an interface or a boundary. Exploiting the analytic properties of the bulk and boundary conformal blocks we have shown how to extract the

\footnotetext{
${ }^{19}$ The integrals and resummations are exactly the same.

${ }^{20}$ In the case when both sides are interacting and $\hat{\gamma}_{0}^{(1 / 2)}$ is zero, i.e. when there are no corrections at order $\sqrt{\epsilon}$, one can use the method in section 2.1 to bootstrap the theory at order $\epsilon$. In such case, removing the branch cuts in $H_{i}$ allows $\hat{\gamma}_{1}^{(1)}$ to be expressed in terms of $\gamma_{\phi}^{(1)}$ and $\hat{\gamma}_{0}^{(1)}$.
} 
CFT data from the bootstrap equation obtained from the two-point correlation function of bulk scalar operators. This is illustrated in the context of perturbative WF theory. We have shown that the bootstrap equation upto the leading order in the $\epsilon$-expansion contains only a few conformal blocks in either channel. This allows us to compute the OPE coefficients at the next order in epsilon. The primary model we studied is the ICFT near four dimensions with quartic interactions where we fixed the OPE coefficients in terms of the anomalous dimensions of the operators in the spectrum. We computed the two-point correlator $\langle\phi \phi\rangle$ upto $\mathcal{O}\left(\epsilon^{2}\right)$. We have shown how to constrain the CFT data further by using the e.o.m. on the two-point correlator which is summarised in 3.3. This is discussed in the context of $\mathrm{RG}$ domain wall when one side of the interface is free. We also studied the CFT near six dimensions with cubic interactions in the presence of a boundary with Dirichlet/Neumann b.c., or an interface, where we fixed the coefficients as well as the correlator upto $\mathcal{O}(\epsilon)$ or $\mathcal{O}(\sqrt{\epsilon})$ respectively. At higher orders in $\epsilon$ there are infinite number of new operators in the bootstrap equation. Hence there is a possibility of having degenerate operators in both the channels which implies that the OPE coefficients can contain contributions from multiple operators. Then one needs to solve the mixing problem and disentangle the operators to go to higher orders in $\epsilon$.

There are several future directions that one can pursue. It would be interesting to apply this approach to compute correlators of composite correlators. For example one can study $\left\langle\phi^{2} \phi^{2}\right\rangle$ in the $\epsilon$-expansion. This is known upto $\mathcal{O}(\epsilon)$ [2], and already in the free theory its block decompositions consists of infinitely many operators in both channels [3]. It would therefore be interesting to develop bootstrap techniques to compute this correlator at the next order.

There are other theories where one can apply this method. One can consider $O(N)$ vector models at large $N$ using non-linear $\sigma$-model, or study theories with fermions, e.g. the large $N$ Gross-Neveu model or the Gross-Neveu-Yukawa model. One can also generalize the techniques of this paper to lower dimensional defects.

It would be interesting to study the two-point function of spinning operators in a CFT with a boundary or defect $[20,30,43]$. For example, one can study the bootstrap techniques to the current and stress-energy two-point functions. Their block decompositions were studied in $[21,44]$. We hope to report on this in future.

\section{Acknowledgments}

We thank Agnese Bissi, Hans Werner Diehl, Pedro Liendo, Marco Meineri and Emilio Trevisani for useful discussions. We also thank Agnese Bissi, Tobias Hansen and Marco Meineri for comments on the draft. This research received funding from the Knut and Alice Wallenberg Foundation grant KAW 2016.0129 and the VR grant 2018-04438.

\section{A Boundary conditions in an ICFT}

In this appendix we motivate our choice of b.c.'s at (3.1) used in the ICFT we consider. This discussion will be a generalization of the b.c.'s in two-dimensions [45], and it was briefly discussed in [21]. 
On the interface, there will be a pseudo stress-energy (SE) tensor $\hat{\tau}^{a b}$ with $a, b \in\{1, \ldots, d-1\}$. Its parallel derivative $\partial_{\|}^{b} \hat{\tau}^{b a}$ is the interface limit of the $T^{a \perp}$ component of the bulk SE tensor. This operator measures the energy emitted or absorbed by the interface. Since there is one bulk SE tensor on each side of the interface: $T_{+}^{\mu \nu}$ and $T_{-}^{\mu \nu}$ with

$\mu, \nu \in\{1, \ldots, d\}$, we identify them on the interface, giving us the following b.c.

$$
\partial_{\|}^{b} \hat{\tau}^{b a}=\hat{T}_{+}^{a \perp}=\hat{T}_{-}^{a \perp} .
$$

In case of a fundamental scalar (3.9), the $T_{ \pm}^{a \perp}$-component of the SE tensor (with correction) is given $b^{21}$

$$
T_{ \pm}^{a \perp}=\partial^{a} \phi_{ \pm}^{i_{ \pm}} \partial^{\perp} \phi_{ \pm}^{i_{ \pm}}-\zeta \partial^{a} \partial^{\perp}\left(\phi_{ \pm}^{i_{ \pm}}\right)^{2}+\mathcal{O}(\lambda), \quad \zeta=\frac{d-2}{4(d-1)}, \quad i_{ \pm} \in\left\{1, \ldots, N_{ \pm}\right\} .
$$

Here we let the scalars on the two sides of the interface have different amount of flavours, with $N_{+} \geq N_{-}$, and the $\mathcal{O}(\lambda)$-terms are corrections from bulk interactions. In the interface limit we also pick up corrections from interface interactions $\hat{\lambda}$

$$
\lim _{z \rightarrow 0} T_{ \pm}^{a \perp}=\lim _{z \rightarrow 0}\left(\partial^{a} \phi_{ \pm}^{i_{ \pm}} \partial^{\perp} \phi_{ \pm}^{i_{ \pm}}\right)-\zeta \partial^{a} \lim _{z \rightarrow 0}\left(\partial^{\perp}\left(\phi_{ \pm}^{i_{ \pm}}\right)^{2}\right)+\mathcal{O}(\lambda, \hat{\lambda})
$$

Given this, one solution to the b.c. equation (A.1) is to relate the fundamental field and its normal derivative for the first $N_{-}$scalars at the interface, and consider reflective b.c.'s for the excessive scalars (i.e. either Dirichlet and Neumann b.c.'s similar to those in a BCFT) ${ }^{22}$

$$
\begin{array}{llll}
\lim _{z \rightarrow 0} \phi_{+}^{i}=\lim _{z \rightarrow 0} \phi_{-}^{i}, & \lim _{z \rightarrow 0} \partial_{\perp} \phi_{+}^{i}=\lim _{z \rightarrow 0} \partial_{\perp} \phi_{-}^{i}, & & i \in\left\{1, \ldots, N_{-}\right\}, \\
\lim _{z \rightarrow 0} \phi_{+}^{m}=0, \quad \text { or }, & \lim _{z \rightarrow 0} \partial_{\perp} \phi_{+}^{m}=0, & m \in\left\{1, \ldots, N_{+}-N_{-}\right\} .
\end{array}
$$

If we have $N_{+}>N_{-}$in above b.c.'s, we have reduced the $O\left(N_{+}\right) \times O\left(N_{-}\right)$ICFT that we started studying into a $O\left(N_{-}\right) \times O\left(N_{-}\right)$ICFT and a $O\left(N_{+}-N_{-}\right)$BCFT (assuming there are no interactions that mix these two systems). These are two systems that one can study separately. Bootstrap methods in BCFTs with $O(N)$ symmetry was studied in [12]. In this paper we study bootstrap methods in the other system, i.e. an ICFT with $O(N) \times O(N)$ symmetry. Hence we consider the amount of flavours to be the same on both sides of the interface in section 3. In such case, we have the b.c.'s at (3.1).

\section{B The $\epsilon$-expansion of boundary conformal blocks}

Here we explain how we $\epsilon$-expand the two boundary blocks $\mathcal{G}_{\text {boe }}\left(\hat{\Delta}_{0}, \xi\right)$ and $\mathcal{G}_{\text {boe }}\left(\hat{\Delta}_{1}, \xi\right)$ using the algorithm in [28]. The main idea is to use the following integral representation for the hypergeometric functions, and $\epsilon$-expand its integrand before performing the integration

$$
{ }_{2} F_{1}(a, b, c, z)=\frac{\Gamma_{c}}{\Gamma_{b} \Gamma_{c-b}} \int_{0}^{1} d t \frac{t^{b-1}(1-t)^{c-b-1}}{(1-t z)^{a}} .
$$

\footnotetext{
${ }^{21}$ This can be seen by varying the action w.r.t. the metric, see e.g. [4].

${ }^{22}$ One can see this by studying the boundary limit of $T_{ \pm}^{a \perp}$ inserted into a correlator, e.g. $\left\langle T_{ \pm}^{a \perp}(x) \hat{O}^{2}(y)\right\rangle$, where $\hat{O}^{2}$ is a composite operator on the interface.
} 
However, the $\epsilon$-expansion and the integral only commutes when the integral converges, which occurs when $\left.c\right|_{\epsilon=0}>\left.b\right|_{\epsilon=0}>0$. To guarantee this we will use the following recurrence relations for the hypergeometric function, and use the above integral representation for each term

$$
\begin{aligned}
{ }_{2} F_{1}(a, b ; c ; z)= & -\frac{z(a+b-2 c-1)+c}{c(z-1)}{ }_{2} F_{1}(a, b ; c+1 ; z) \\
& -\frac{z(a-c-1)(b-c-1)}{c(c+1)(z-1)}{ }_{2} F_{1}(a, b ; c+2 ; z) .
\end{aligned}
$$

After expanding in $\epsilon$, we need to integrate over $t$. To perform this integration, we use the Mathematica command HypExpInt [28], which evaluates the integral

$$
\operatorname{Hyp} \operatorname{ExpInt}\left(a_{1}, a_{2}, a_{3}, a_{4}, a_{5}, z\right)=\int_{0}^{1} d t \frac{t^{a_{1}} \log (t)^{a_{2}} \log (1-t)^{a_{3}} \log (1-t z)^{a_{4}}}{(t z-1)^{a_{5}}} .
$$

This yields the following expansions of hypergeometric functions in $\mathcal{G}_{\text {boe }}\left(\hat{\Delta}_{0}, \xi\right)$ and $\mathcal{G}_{\text {boe }}\left(\hat{\Delta}_{1}, \xi\right)$

$$
\begin{aligned}
{ }_{2} F_{1} & \left(\frac{1}{2}(-2 \alpha-1) \epsilon+\hat{\gamma}_{0}^{(2)} \epsilon^{2}+1, \hat{\gamma}_{0}^{(2)} \epsilon^{2}-\alpha \epsilon ; 2 \hat{\gamma}_{0}^{(2)} \epsilon^{2}-2 \alpha \epsilon ; z\right) \\
= & \frac{z-2}{2(z-1)}+\epsilon\left(\frac{\alpha}{2}+\frac{1}{4-4 z}\right) \log (1-z)+\epsilon^{2}\left(\frac{(1-2 \alpha) \alpha z \operatorname{Li}_{2}(z)}{4(z-1)}\right. \\
& \left.+\left(\frac{\alpha}{8}+\frac{1}{16-16 z}\right) \log ^{2}(1-z)-\frac{1}{2} \hat{\gamma}_{0}^{(2)} \log (1-z)\right) \\
{ }_{2} F_{1}\left(2-\left(\frac{1}{2}+\alpha\right) \epsilon+\hat{\gamma}_{1}^{(2)} \epsilon^{2}, 1-\alpha \epsilon+\hat{\gamma}_{1}^{(2)} \epsilon^{2}, 2-2 \alpha \epsilon+2 \hat{\gamma}_{1}^{(2)} \epsilon^{2}, z\right) & \\
= & \frac{1}{1-z}+\epsilon\left(\frac{\alpha-\frac{1}{2}}{z-1}+\frac{\left(\alpha+\alpha(-z)-\frac{1}{2}\right) \log (1-z)}{(z-1) z}\right) \\
& +\epsilon^{2}\left(\frac{\left(\alpha-\frac{1}{2}\right) \alpha(z-2) \operatorname{Li}{ }_{2}(z)}{(z-1) z}+\frac{(-2 \alpha+2 \alpha z+1) \log ^{2}(1-z)}{8 z-8 z^{2}}\right. \\
& \left.+\frac{-4 \alpha^{2}+4 \hat{\gamma}_{1}^{(2)}+1}{4-4 z}+\frac{\log (1-z)\left(4 \alpha^{2}(z-1)-2 \alpha(z-2)+4 \hat{\gamma}_{1}^{(2)}(z-1)-1\right)}{4(z-1) z}\right) .
\end{aligned}
$$

\section{Resummation techniques}

In this appendix we describe the different techniques we use for resuming the bulk and boundary-channels (using Mathematica).

\section{C.1 Expansion in $\xi$}

We use this technique when resuming the bulk-channel in (2.3).

1. Use first the integral representation (B.1) for the hypergeometric functions.

2. Resum the blocks. 
3. Expand the integrand around $\xi=0$. This means that after we have performed the integration, we have to resum the expansion in $\xi$. For each power in $\xi$ we have polynomials in the integration parameter.

4. Integrate the polynomials.

5. Find a general term in the expansion of $\xi$.

6. Resum the expansion in $\xi$. This is the final result.

\section{C.2 Differentiate with respect to $\xi$ and simplify using an ansatz}

This method is used when resuming even and odd operators in the boundary-channel in (2.3).

1. Use first the integral representation (B.1) for the hypergeometric functions.

2. Resum the blocks.

3. For odd blocks $\left(\hat{\Delta}=2 m+1, m \in \mathbb{Z}_{\geq 0}\right)$, differentiate with respect to $\xi$. This is only useful when the derivative is on a simpler form as the original integrand, e.g. when taking the derivative removes dilogarithmic terms.

4. Perform the integration from (B.1).

5. If a derivative w.r.t. $\xi$ was taken, perform an indefinite integrate over $\xi$. This will yield an integration constant that we need to fix.

6. The integration constant is fixed by expanding our result around large $\xi \gg 1$. Compare this with the expansion of the original integrand in step 2, where we only integrate the term at lowest order $\left(\xi^{0}\right)$.

7. This is the final result. Near four dimensions it may be a cumbersome expression that contains several different dilogarithms. One way to simplify it is to make the following ansatz (with the correct branch cuts only along $\xi \in(-1,0)$ ) for your result

$$
\begin{aligned}
f(\xi)= & A_{1}+A_{2} \frac{\xi}{\xi+1}+\left(A_{3}+A_{4} \frac{\xi}{\xi+1}\right) \log \left(\frac{\xi}{\xi+1}\right) \\
& +\left(A_{5}+A_{6} \frac{\xi}{\xi+1}\right) \log ^{2}\left(\frac{\xi}{\xi+1}\right)+\left(A_{7}+A_{8} \frac{\xi}{\xi+1}\right) \operatorname{Li}_{2}\left(-\frac{1}{\xi}\right) .
\end{aligned}
$$

8. The coefficients $A_{i}, i \in\{1, \ldots, 8\}$ in our ansatz can be found by comparing the expansions around large $\xi$ with that of the final result from the previous step. Some of the dilogarithms can be expanded after using the following identity

$$
\operatorname{Li}_{2}(z)=\frac{\pi^{2}}{6}-\operatorname{Li}_{2}(1-z)-\log (z) \log (1-z) .
$$

Open Access. This article is distributed under the terms of the Creative Commons Attribution License (CC-BY 4.0), which permits any use, distribution and reproduction in any medium, provided the original author(s) and source are credited. 


\section{References}

[1] F. Gliozzi, P. Liendo, M. Meineri and A. Rago, Boundary and Interface CFTs from the Conformal Bootstrap, JHEP 05 (2015) 036 [arXiv: 1502.07217] [INSPIRE].

[2] D.M. McAvity and H. Osborn, Conformal field theories near a boundary in general dimensions, Nucl. Phys. B 455 (1995) 522 [cond-mat/9505127] [INSPIRE].

[3] P. Liendo, L. Rastelli and B.C. van Rees, The Bootstrap Program for Boundary CFT $T_{d}$, JHEP 07 (2013) 113 [arXiv: 1210.4258] [inSPIRE].

[4] V. Procházka and A. Söderberg, Composite operators near the boundary, JHEP 03 (2020) 114 [arXiv: 1912.07505] [INSPIRE].

[5] C.P. Herzog and N. Kobayashi, The $O(N)$ model with $\phi^{6}$ potential in $\mathbb{R}^{2} \times \mathbb{R}^{+}$, JHEP 09 (2020) 126 [arXiv : 2005. 07863] [inSPIRE].

[6] P. Dey, T. Hansen and M. Shpot, Operator expansions, layer susceptibility and two-point functions in BCFT, JHEP 12 (2020) 051 [arXiv:2006.11253] [INSPIRE].

[7] H.W. Diehl, Why boundary conditions do not generally determine the universality class for boundary critical behavior, Eur. Phys. J. B 93 (2020) 195 [arXiv:2006.15425] [INSPIRE].

[8] S. Giombi, H. Khanchandani and X. Zhou, Aspects of CFTs on Real Projective Space, J. Phys. A 54 (2021) 024003 [arXiv:2009.03290] [InSPIRE].

[9] C. Behan, L. Di Pietro, E. Lauria and B.C. Van Rees, Bootstrapping boundary-localized interactions, JHEP 12 (2020) 182 [arXiv:2009.03336] [INSPIRE].

[10] A. Gimenez-Grau, P. Liendo and P. van Vliet, Superconformal boundaries in $4-\epsilon$ dimensions, JHEP 04 (2021) 167 [arXiv:2012.00018] [INSPIRE].

[11] V. Procházka and A. Söderberg, Spontaneous symmetry breaking in free theories with boundary potentials, arXiv:2012.00701 [INSPIRE].

[12] A. Bissi, T. Hansen and A. Söderberg, Analytic Bootstrap for Boundary CFT, JHEP 01 (2019) 010 [arXiv: 1808.08155] [inSPIRE].

[13] A. Kaviraj and M.F. Paulos, The Functional Bootstrap for Boundary CFT, JHEP 04 (2020) 135 [arXiv: 1812.04034] [INSPIRE].

[14] D. Mazáč, L. Rastelli and X. Zhou, An analytic approach to BCFT $T_{d}$, JHEP 12 (2019) 004 [arXiv: 1812.09314] [INSPIRE].

[15] S. Rychkov and Z.M. Tan, The $\epsilon$-expansion from conformal field theory, J. Phys. A 48 (2015) 29FT01 [arXiv: 1505.00963] [INSPIRE].

[16] S. Yamaguchi, The $\epsilon$-expansion of the codimension two twist defect from conformal field theory, PTEP 2016 (2016) 091B01 [arXiv:1607.05551] [INSPIRE].

[17] A. Söderberg, Anomalous Dimensions in the WF $O(N)$ Model with a Monodromy Line Defect, JHEP 03 (2018) 058 [arXiv: 1706. 02414] [INSPIRE].

[18] S. Giombi and H. Khanchandani, CFT in AdS and boundary RG flows, JHEP 11 (2020) 118 [arXiv:2007.04955] [INSPIRE].

[19] M.A. Shpot, Boundary conformal field theory at the extraordinary transition: The layer susceptibility to $O(\varepsilon)$, JHEP 01 (2021) 055 [arXiv: 1912.03021] [INSPIRE].

[20] E. Lauria, M. Meineri and E. Trevisani, Radial coordinates for defect CFTs, JHEP 11 (2018) 148 [arXiv: 1712.07668] [INSPIRE]. 
[21] C.P. Herzog and K.-W. Huang, Boundary Conformal Field Theory and a Boundary Central Charge, JHEP 10 (2017) 189 [arXiv:1707.06224] [INSPIRE].

[22] A.J. Bray and M.A. Moore, Critical Behavior of a Semiinfinite System: n Vector Model in the Large $N$ Limit, Phys. Rev. Lett. 38 (1977) 735 [InSPIRE].

[23] K. Symanzik, Schrödinger Representation and Casimir Effect in Renormalizable Quantum Field Theory, Nucl. Phys. B 190 (1981) 1 [inSPIRE].

[24] T.W. Burkhardt and E. Eisenriegler, Critical phenomena near free surfaces and defect planes, Phys. Rev. B 24 (1981) 1236.

[25] E. Eisenriegler and T.W. Burkhardt, Universal and nonuniversal critical behavior of the $n$-vector model with a defect plane in the limit $n \rightarrow \infty$, Phys. Rev. B 25 (1982) 3283.

[26] H.W. Diehl, S. Dietrich and E. Eisenriegler, Universality, irrelevant surface operators, and corrections to scaling in systems with free surfaces and defect planes, Phys. Rev. B 27 (1983) 2937 [INSPIRE].

[27] E. Wong and I. Affleck, Tunneling in quantum wires: A Boundary conformal field theory approach, Nucl. Phys. B 417 (1994) 403 [cond-mat/9311040] [InSPIRE].

[28] T. Huber and D. Maître, HypExp: A Mathematica package for expanding hypergeometric functions around integer-valued parameters, Comput. Phys. Commun. 175 (2006) 122 [hep-ph/0507094] [INSPIRE].

[29] K.G. Wilson, Quantum field theory models in less than four-dimensions, Phys. Rev. D 7 (1973) 2911 [INSPIRE].

[30] M. Billò, V. Gonçalves, E. Lauria and M. Meineri, Defects in conformal field theory, JHEP 04 (2016) 091 [arXiv: 1601.02883] [INSPIRE].

[31] I. Lawrie and S. Sarlbach, Tricriticality phase transitions and critical phenomena, vol. 9, ed. C. Domb and J.L. Lebowitz, London Academic (1984).

[32] J.S. Reeve and A.J. Guttmann, Critical behavior of the n-vector model with a free surface, Phys. Rev. Lett. 45 (1980) 1581.

[33] J.S. Reeve, Renormalisation group calculation of the critical exponents of the special transition in semi-infinite systems, Phys. Lett. A 81 (1981) 237.

[34] H. Diehl and S. Dietrich, Field-theoretical approach to static critical phenomena in semi-infinite systems, Z. Phys. B 43 (1981) 281.

[35] W. Speth, Tricritical phase transitions in semi-infinite systems, Z. Phys. B 51 (1983) 361.

[36] H.W. Diehi and E. Eisenriegler, Walks, polymers, and other tricritical systems in the presence of walls or surfaces, EPL 4 (1987) 709.

[37] M.E. Fisher, Yang-Lee Edge Singularity and phi**3 Field Theory, Phys. Rev. Lett. 40 (1978) 1610 [INSPIRE].

[38] H.K. Janssen and A. Lyssy, Adsorption-transition of branched polymers at surfaces: Superuniversality of the crossover exponent, EPL 29 (1995) 25.

[39] J.P. Carton, Surface effects and percolation: the repulsive case, J. Phys. Lett. 41 (1980) 175.

[40] H.W. Diehl and P.M. Lam, Semi-infinite Potts model and percolation at surfaces, Z. Phys. B 74 (1989) 395. 
[41] R.G. Priest and T.C. Lubensky, Critical properties of two tensor models with application to the percolation problem, Phys. Rev. B 13 (1976) 4159 [INSPIRE].

[42] D.J. Amit, Renormalization of the Potts Model, J. Phys. A 9 (1976) 1441 [InSPIRE].

[43] E. Lauria, M. Meineri and E. Trevisani, Spinning operators and defects in conformal field theory, JHEP 08 (2019) 066 [arXiv:1807.02522] [INSPIRE].

[44] C.P. Herzog and A. Shrestha, Two point functions in defect CFTs, JHEP 04 (2021) 226 [arXiv: 2010.04995] [INSPIRE].

[45] C. Bachas, J. de Boer, R. Dijkgraaf and H. Ooguri, Permeable conformal walls and holography, JHEP 06 (2002) 027 [hep-th/0111210] [INSPIRE]. 